\title{
Degeneration of multiple Euler products
}

\author{
by
}

\author{
Hirotaka Akatsuka (Tokyo)
}

1. Introduction. Let

$$
Z_{j}(s) \cong \prod_{\varrho \in \mathbb{C}}((s-\varrho))^{m_{j}(\varrho)}:=\exp \left[-\left.\frac{\partial}{\partial w} \sum_{\varrho \in \mathbb{C}} \frac{m_{j}(\varrho)}{(s-\varrho)^{w}}\right|_{w=0}\right]
$$

be meromorphic functions expressed as regularized products, where $f(s) \cong$ $g(s)$ means that $f(s)=e^{Q(s)} g(s)$ for some $Q(s) \in \mathbb{C}[s]$. Then their absolute tensor product is defined as

$$
Z_{1}(s) \otimes \cdots \otimes Z_{r}(s):=\prod_{\varrho_{1}, \ldots, \varrho_{r} \in \mathbb{C}}\left(\left(s-\varrho_{1}-\cdots-\varrho_{r}\right)\right)^{m\left(\varrho_{1}, \ldots, \varrho_{r}\right)},
$$

where

$$
m\left(\varrho_{1}, \ldots, \varrho_{r}\right):=m_{1}\left(\varrho_{1}\right) \cdots m_{r}\left(\varrho_{r}\right) \times \begin{cases}1 & \text { if } \operatorname{Im}\left(\varrho_{1}\right), \ldots, \operatorname{Im}\left(\varrho_{r}\right) \geq 0, \\ (-1)^{r-1} & \text { if } \operatorname{Im}\left(\varrho_{1}\right), \ldots, \operatorname{Im}\left(\varrho_{r}\right)<0, \\ 0 & \text { otherwise. }\end{cases}
$$

The absolute tensor product was originally investigated by Kurokawa [K]. We refer to Manin [M] for an excellent survey.

Suppose that for any $j \in\{1, \ldots, r\}, Z_{j}(s)$ has an Euler product expression

$$
Z_{j}(s)=\prod_{p \in P_{j}} H_{p}^{j}\left(N_{j}(p)^{-s}\right)
$$

with $H_{p}^{j}(T) \in 1+T \mathbb{C}[[T]]$. Then we expect that $Z_{1}(s) \otimes \cdots \otimes Z_{r}(s)$ has a multiple Euler product expression, that is,

$$
Z_{1}(s) \otimes \cdots \otimes Z_{r}(s) \cong \prod_{\left(p_{1}, \ldots, p_{r}\right) \in P_{1} \times \cdots \times P_{r}} H_{\left(p_{1}, \ldots, p_{r}\right)}\left(N_{1}\left(p_{1}\right)^{-s}, \ldots, N_{r}\left(p_{r}\right)^{-s}\right)
$$

for some $H_{\left(p_{1}, \ldots, p_{r}\right)}\left(T_{1}, \ldots, T_{r}\right) \in \mathbb{C}\left[\left[T_{1}, \ldots, T_{r}\right]\right]$ with constant term 1 . We expect $H_{\left(p_{1}, \ldots, p_{r}\right)}$ to have a degenerate form when $N_{j}\left(p_{j}\right)=N_{k}\left(p_{k}\right)$ for some $j, k(j \neq k)$.

2000 Mathematics Subject Classification: Primary 11M41.

The author is supported by JSPS Research Fellowships for Young Scientists. 
Recall that the Hasse zeta function for a commutative ring $A$ is defined as

$$
\zeta(s, A):=\prod_{\mathfrak{m}}(1-N(\mathfrak{m}))^{-1},
$$

where $\mathfrak{m}$ runs through all maximal ideals of $A$ and $N(\mathfrak{m}):=\#(A / \mathfrak{m})$.

Absolute tensor products of Hasse zeta functions for finite fields were investigated in [KK1], $[\mathrm{A}]$ and $[\mathrm{KW}]$. We have

THEOREM 1.1. Let $p_{1}, \ldots, p_{r}$ be distinct prime numbers. Then for $\operatorname{Re}(s)$ $>0$ we have

$$
\zeta\left(s, \mathbb{F}_{p_{1}}\right) \otimes \cdots \otimes \zeta\left(s, \mathbb{F}_{p_{r}}\right) \cong \exp \left[\sum_{j=1}^{r} \sum_{m=1}^{\infty} \frac{c_{m}\left(p_{j} ; p_{1}, \stackrel{\stackrel{j}{v}}{m}, p_{r}\right)}{m} p_{j}^{-m s}\right],
$$

where

$$
c_{m}\left(p_{1} ; p_{2}, \ldots, p_{r}\right):=\frac{(-1)^{r+1}}{\prod_{k=2}^{r}\left(1-e\left(m \frac{\log p_{1}}{\log p_{k}}\right)\right)} \quad\left(e(x):=e^{2 \pi i x}\right)
$$

and $\stackrel{j}{\vee}$ means removing the $j$ th component.

From this result we see that $\zeta\left(s, \mathbb{F}_{p_{1}}\right) \otimes \cdots \otimes \zeta\left(s, \mathbb{F}_{p_{r}}\right)$ has a multiple Euler product expression when $p_{1}, \ldots, p_{r}$ are distinct prime numbers.

On the other hand, when $p_{j}=p_{k}$ for some $j, k(j \neq k)$ (that is, in the case where we expect a degeneration of the multiple Euler product), $[\mathrm{K}]$ essentially asserts the following:

TheOREM 1.2 ([K, Theorem 1]). Let $p$ be a prime number and $r$ be a positive integer. Then for $\operatorname{Re}(s)>0$ we have

$$
\zeta\left(s, \mathbb{F}_{p}\right)^{\otimes r} \cong \exp \left[\sum_{k=1}^{r} \frac{1}{(-2 \pi i)^{k-1}} g_{r}^{(k-1)}\left(-\frac{s \log p}{2 \pi i}\right) \operatorname{Li}_{k}\left(p^{-s}\right)\right],
$$

where

$$
g_{r}(x):= \begin{cases}\frac{(x-1)(x-2) \cdots(x-(r-1))}{(r-1) !} & \text { if } r \geq 2 \\ 1 & \text { if } r=1\end{cases}
$$

$g_{r}^{(k)}$ denotes the kth derivative of $g_{r}$ and

$$
\operatorname{Li}_{k}(x):=\sum_{n=1}^{\infty} \frac{x^{n}}{n^{k}} \quad(|x|<1) .
$$

The aim of this paper is to investigate the degeneration of multiple Euler products more generally. We calculate degenerate forms of the multiple Euler product for $\zeta\left(s, \mathbb{F}_{p}\right)^{\otimes M} \otimes \zeta\left(s, \mathbb{F}_{q}\right)^{\otimes N}$. The main result is 
TheOREM 1. Let $p, q$ be distinct prime numbers and $M, N$ be positive integers. Then for $\operatorname{Re}(s)>0$ we have

$$
\begin{aligned}
\zeta\left(s, \mathbb{F}_{p}\right)^{\otimes M} \otimes & \zeta\left(s, \mathbb{F}_{q}\right)^{\otimes N} \\
\cong & \exp \left[\left.i \log p \cdot \sum_{n=1}^{\infty} g_{M}\left(\frac{\log p}{2 \pi} \frac{\partial}{\partial t}\right) P_{N}\left(t ; i s ; \frac{2 \pi}{\log q}\right)\right|_{t=i n \log p}\right. \\
& \left.+\left.i \log q \cdot \sum_{n=1}^{\infty} g_{N}\left(\frac{\log q}{2 \pi} \frac{\partial}{\partial t}\right) P_{M}\left(t ; i s ; \frac{2 \pi}{\log p}\right)\right|_{t=i n \log q}\right]
\end{aligned}
$$

where

$$
P_{M}(t ; z ; \omega):=\frac{e^{t z}}{t\left(e^{t \omega}-1\right)^{M}}
$$

For the proof we use the method of [KK1], showing a corresponding expression for the multiple sine function. We now recall the construction of the multiple sine function from [KK2]. First we define the Barnes multiple zeta function as

$$
\zeta_{r}(s, z, \underline{\omega}):=\sum_{n_{1}, \ldots, n_{r}=0}^{\infty}\left(n_{1} \omega_{1}+\cdots+n_{r} \omega_{r}+z\right)^{-s}
$$

for $\underline{\omega}=\left(\omega_{1}, \ldots, \omega_{r}\right) \in\left(\mathbb{R}_{>0}\right)^{r}$. This series converges absolutely for $\operatorname{Re}(s)$ $>r$. This function admits a meromorphic continuation to all $s \in \mathbb{C}$ and is holomorphic at $s=0$. The multiple gamma function $\Gamma_{r}(z, \underline{\omega})$ and the multiple sine function $S_{r}(z, \underline{\omega})$ are defined as

$$
\begin{aligned}
\Gamma_{r}(z, \underline{\omega}) & :=\exp \left[\left.\frac{\partial}{\partial s} \zeta_{r}(s, z, \underline{\omega})\right|_{s=0}\right], \\
S_{r}(z, \underline{\omega}) & :=\Gamma_{r}(z, \underline{\omega})^{-1} \Gamma_{r}(|\underline{\omega}|-z, \underline{\omega})^{(-1)^{r}},
\end{aligned}
$$

where $|\underline{\omega}|:=\omega_{1}+\cdots+\omega_{r}$.

To deal with the convergence problem, we introduce some notions of Diophantine approximation theory. We say a real number $a$ is generic if

$$
\lim _{m \rightarrow \infty}\|m a\|^{1 / m}=1,
$$

where $\|x\|:=\min \{|x-n|: n \in \mathbb{Z}\}$. For example, $a=\frac{\log x}{\log y}$ is generic for any $x, y \in \overline{\mathbb{Q}} \cap \mathbb{R}_{>0}$ such that $a \notin \mathbb{Q}[\mathrm{B}$, Theorem 3.1].

The corresponding expression for the multiple sine function is as follows:

Theorem 2. Let $M, N$ be positive integers and $\omega_{1}, \omega_{2}$ be positive real numbers such that $\omega_{1} / \omega_{2}$ and $\omega_{2} / \omega_{1}$ are generic. Then for $\operatorname{Im}(z)>0$ we 
have

$$
\begin{aligned}
S_{r}(z, \underline{\omega}) \cong & \exp \left[\left.\frac{2 \pi i(-1)^{r}}{\omega_{1}} \sum_{n=1}^{\infty} g_{M}\left(\frac{1}{\omega_{1}} \frac{\partial}{\partial t}\right) P_{N}\left(t ; z ; \omega_{2}\right)\right|_{t=2 \pi i n / \omega_{1}}\right. \\
& \left.+\left.\frac{2 \pi i(-1)^{r}}{\omega_{2}} \sum_{n=1}^{\infty} g_{N}\left(\frac{1}{\omega_{2}} \frac{\partial}{\partial t}\right) P_{M}\left(t ; z ; \omega_{1}\right)\right|_{t=2 \pi i n / \omega_{2}}\right],
\end{aligned}
$$

where $r:=M+N$ and $\underline{\omega}:=(\underbrace{\omega_{1}, \ldots, \omega_{1}}_{M \text { copies }}, \underbrace{\omega_{2}, \ldots, \omega_{2}}_{N \text { copies }})$.

By the relation between $\zeta\left(s, \mathbb{F}_{p}\right)^{\otimes M} \otimes \zeta\left(s, \mathbb{F}_{q}\right)^{\otimes N}$ and $S_{r}(z, \underline{\omega})$ (see Lemma 4.1 below) Theorem 2 implies Theorem 1 directly. The key to the proof of Theorem 2 is the following explicit formula, which we call the signatured Poisson summation formula with weight:

Theorem 3. Let $m_{1}, m_{2}$ be positive integers and put $m:=m_{1}+m_{2}$. Suppose that a function $H$ satisfies the following assumptions:

(T1) There exists $A>0$ such that $H$ is holomorphic in the region including the strip

$$
S:=\{t \in \mathbb{C}:|\operatorname{Im}(t)| \leq A\} .
$$

(T2) There exists $\delta>0$ such that

$$
H(t) \ll(|t|+1)^{-m-\delta} \quad \text { for } t \in S,
$$

where the implied constant depends only on $m, A, \delta$.

(T3) $H(-t)=(-1)^{m-1} H(t)$.

Let $\omega_{1}, \omega_{2}$ be positive real numbers such that $\omega_{1} / \omega_{2}$ and $\omega_{2} / \omega_{1}$ are generic. Then

$$
\begin{aligned}
& \sum_{n_{1}, n_{2} \geq 0} \varepsilon_{n_{1}, n_{2}} n_{1}^{m_{1}-1} n_{2}^{m_{2}-1} H\left(n_{1} \omega_{1}+n_{2} \omega_{2}\right) \\
& =\frac{1}{(-2 \pi i)^{m-1}} \frac{1}{\omega_{1}^{m_{1}} \omega_{2}^{m_{2}}}\left(\frac{1}{2} \frac{\left(m_{1}-1\right) !\left(m_{2}-1\right) !}{(m-1) !} \widehat{H}^{(m-1)}(0)\right. \\
& \quad+\sum_{k=0}^{m_{1}-1}(-1)^{k}\left(\begin{array}{c}
m_{1}-1 \\
k
\end{array}\right)\left(m_{2}+k-1\right) ! \\
& \quad \times \sum_{\substack{n_{1}, n_{2} \geq 0 \\
\left(n_{1}, n_{2}\right) \neq(0,0)}} \varepsilon_{n_{1}, n_{2}}\left(\frac{1}{\left(\frac{n_{1}}{\omega_{1}}-\frac{n_{2}}{\omega_{2}}\right)^{m_{2}+k}}+\frac{1}{\left(\frac{n_{1}}{\omega_{1}}+\frac{n_{2}}{\omega_{2}}\right)^{m_{2}+k}}\right) \widehat{H}^{\left(m_{1}-k-1\right)}\left(\frac{n_{1}}{\omega_{1}}\right)
\end{aligned}
$$




$$
\begin{aligned}
& +\sum_{k=0}^{m_{2}-1}(-1)^{k}\left(\begin{array}{c}
m_{2}-1 \\
k
\end{array}\right)\left(m_{1}+k-1\right) ! \\
& \left.\times \sum_{\substack{n_{1}, n_{2} \geq 0 \\
\left(n_{1}, n_{2}\right) \neq(0,0)}} \varepsilon_{n_{1}, n_{2}}\left(\frac{1}{\left(\frac{n_{2}}{\omega_{2}}-\frac{n_{1}}{\omega_{1}}\right)^{m_{1}+k}}+\frac{1}{\left(\frac{n_{2}}{\omega_{2}}+\frac{n_{1}}{\omega_{1}}\right)^{m_{1}+k}}\right) \widehat{H}^{\left(m_{2}-k-1\right)}\left(\frac{n_{2}}{\omega_{2}}\right)\right),
\end{aligned}
$$

where

$$
\varepsilon_{n_{1}, n_{2}}:=\varepsilon_{n_{1}} \varepsilon_{n_{2}}, \quad \varepsilon_{n}:=\left\{\begin{array}{ll}
1 / 2 & \text { if } n=0, \\
1 & \text { if } n \neq 0,
\end{array} \quad \widehat{H}(x):=\int_{-\infty}^{\infty} H(t) e(-t x) d t .\right.
$$

In view of Theorem 2 and the expressions of multiple sine functions corresponding to Theorems 1.1 and 1.2 we conjecture expressions of multiple sine functions for a more general situation.

Conjecture 4. Let $k \in \mathbb{Z}_{\geq 1}$ and $\underline{M}:=\left(M_{1}, \ldots, M_{k}\right) \in\left(\mathbb{Z}_{\geq 1}\right)^{k}$. Put $r:=M_{1}+\cdots+M_{k}$. Suppose that

$$
\underline{\omega}:=(\underbrace{\omega_{1}, \ldots, \omega_{1}}_{M_{1} \text { copies }}, \ldots, \underbrace{\omega_{k}, \ldots, \omega_{k}}_{M_{k} \text { copies }}) \in \mathbb{C}^{r}
$$

satisfies (i) or (ii), where

(i) $\underline{\omega} \in\left(\mathbb{R}_{>0}\right)^{r}$ and $\omega_{j} / \omega_{l}$ are generic for any $j, l(j \neq l)$.

(ii) $0<\arg \left(\omega_{1}\right)<\cdots<\arg \left(\omega_{k}\right)<\pi$.

Then in the region $\arg \left(\omega_{k}\right)<\arg (z)<\arg \left(\omega_{1}\right)+\pi$ we have

$$
\cong \exp \left[\left.2 \pi i(-1)^{r} \sum_{j=1}^{k} \frac{1}{\omega_{j}} \sum_{n=1}^{\infty} g_{M_{j}}\left(\frac{1}{\omega_{j}} \frac{\partial}{\partial t}\right) P\left(t ; z ; \underline{\omega}^{\prime}\langle j\rangle ; \underline{M}\langle j\rangle\right)\right|_{t=2 \pi i n / \omega_{j}}\right]
$$

where $\underline{\omega}^{\prime}:=\left(\omega_{1}, \ldots, \omega_{k}\right) \in \mathbb{C}^{k}, \underline{\omega}^{\prime}\langle j\rangle:=\left(\omega_{1}, \stackrel{j}{j}, \omega_{k}\right) \in \mathbb{C}^{k-1}$ and

$$
P\left(t ; z ;\left(\omega_{2}, \ldots, \omega_{k}\right) ;\left(M_{2}, \ldots, M_{k}\right)\right):= \begin{cases}e^{t z} / t & \text { if } k=1, \\ \frac{e^{t z}}{t \prod_{l=2}^{k}\left(e^{t \omega_{l}}-1\right)^{M_{l}}} & \text { if } k \in \mathbb{Z}_{\geq 2} .\end{cases}
$$

Suppose that Conjecture 4 holds when $\underline{\omega}$ satisfies condition (i). Using the relation between absolute tensor products of $\zeta\left(s, \mathbb{F}_{p}\right)$ and multiple sine functions (see Lemma 4.1), we also conjecture a degenerate form of the multiple Euler product for absolute tensor products of $\zeta\left(s, \mathbb{F}_{p}\right)$ :

Conjecture 5. Let $k$ be a positive integer, $\underline{M}:=\left(M_{1}, \ldots, M_{k}\right) \in$ $\left(\mathbb{Z}_{\geq 1}\right)^{k}$ and $p_{1}, \ldots, p_{k}$ be distinct prime numbers. Put

$$
\underline{\eta}:=\left(\frac{2 \pi}{\log p_{1}}, \ldots, \frac{2 \pi}{\log p_{k}}\right) \in\left(\mathbb{R}_{>0}\right)^{k} .
$$


Then for $\operatorname{Re}(s)>0$ we have

$$
\begin{aligned}
& \zeta\left(s, \mathbb{F}_{p_{1}}\right)^{\otimes M_{1}} \otimes \cdots \otimes \zeta\left(s, \mathbb{F}_{p_{k}}\right)^{\otimes M_{k}} \\
& \cong \exp \left[\left.i \sum_{j=1}^{k} \log p_{j} \sum_{n=1}^{\infty} g_{M_{j}}\left(\frac{\log p_{j}}{2 \pi} \frac{\partial}{\partial t}\right) P(t ; i s ; \underline{\eta}\langle j\rangle ; \underline{M}\langle j\rangle)\right|_{t=i n \log p_{j}}\right] .
\end{aligned}
$$

In Section 2 we prove Theorem 3. Then, in Section 3 we prove Theorem 2 by using Theorem 3. In Section 4 we deduce Theorem 1 from Theorem 2 . In Section 5 we see that Conjectures 4 and 5 are reasonable. In the last Section 6 we prove results needed in Section 2. In Section 6.1 we treat differentiability and estimates of the Fourier transformation. In Section 6.2 we give estimations for generic numbers.

2. Signatured Poisson summation formula with weight. In this section we prove Theorem 3 . Throughout this section, $m_{1}, m_{2}, m, H, A, \delta$, $\omega_{1}, \omega_{2}$ are as in Theorem 3 .

First, we outline the proof. Let $0<\alpha<\min \left\{A / 4, \omega_{1} / 2, \omega_{2} / 2\right\}$ and $N$ be a positive integer. We put $h(t):=H(t / i)$ and

$$
\begin{aligned}
I(\alpha, N):= & \frac{1}{(2 \pi i)^{2}} \int_{\partial D_{\alpha, N}\left(\omega_{1}\right)} \int_{\partial D_{\alpha, N}\left(\omega_{2}\right)} h\left(t_{1}+t_{2}\right) t_{1}^{m_{1}-1} t_{2}^{m_{2}-1} \\
& \times \operatorname{coth}\left(\frac{\pi t_{1}}{\omega_{1}}\right) \operatorname{coth}\left(\frac{\pi t_{2}}{\omega_{2}}\right) d t_{2} d t_{1},
\end{aligned}
$$

where

$$
D_{\alpha, N}(\omega):=\{t \in \mathbb{C}:|\operatorname{Re}(t)|<\alpha,|t|>\alpha, 0<\operatorname{Im}(t)<(N+1 / 2) \omega\}
$$

and the integrals along $\partial D_{\alpha, N}\left(\omega_{j}\right)$ are taken counterclockwise. We calculate

$$
I:=\lim _{\alpha \downarrow 0} \lim _{N \rightarrow \infty} I(\alpha, N)
$$

by two methods. In the first method we use residue calculations and in the second method we use residue calculations and integral calculations.

Proof of Theorem 3. We calculate $I$ by the first method. By the residue theorem used repeatedly, we have

$$
I=-\frac{\omega_{1}^{m_{1}} \omega_{2}^{m_{2}} i^{m}}{\pi^{2}} \sum_{n_{1}, n_{2} \geq 1} n_{1}^{m_{1}-1} n_{2}^{m_{2}-1} H\left(n_{1} \omega_{1}+n_{2} \omega_{2}\right) .
$$

Here (T2) guarantees the absolute convergence of (2.2).

Next, we calculate $I$ by the second method. We decompose $\partial D_{\alpha, N}(\omega)=$ $\bigcup_{j=1}^{4} C_{j}(\alpha, N, \omega)$ with 


$$
\begin{aligned}
& C_{1}(\alpha, N, \omega):=\{\alpha+i t: 0 \leq t \leq(N+1 / 2) \omega\} \\
& C_{2}(\alpha, N, \omega):=\{\sigma+i(N+1 / 2) \omega:-\alpha \leq \sigma \leq \alpha\} \\
& C_{3}(\alpha, N, \omega):=\{-\alpha+i t: 0 \leq t \leq(N+1 / 2) \omega\} \\
& C_{4}(\alpha, N, \omega):=\left\{\alpha e^{i \theta}: 0 \leq \theta \leq \pi\right\}
\end{aligned}
$$

Then

$$
I(\alpha, N)=J_{1}(\alpha, N)+J_{2}(\alpha, N)
$$

where

$$
J_{1}(\alpha, N):=\sum_{j=1}^{3} \sum_{k=1}^{3} I_{j k}(\alpha, N), \quad J_{2}(\alpha, N):=\sum_{(j, k) \in\{1,2,3,4\}^{2} \backslash\{1,2,3\}^{2}} I_{j k}(\alpha, N)
$$

and $I_{j k}(\alpha, N):=(2 \pi i)^{-2} \int_{C_{j}\left(\alpha, N, \omega_{1}\right)} \int_{C_{k}\left(\alpha, N, \omega_{2}\right)} \cdots d t_{2} d t_{1}$ in $(2.1)$.

We first consider $\lim _{\alpha \downarrow} \lim _{N \rightarrow \infty} J_{2}(\alpha, N)$. We have

$$
J_{2}(\alpha, N)=\sum_{k=1}^{4} I_{4 k}(\alpha, N)+\sum_{j=1}^{4} I_{j 4}(\alpha, N)-I_{44}(\alpha, N) .
$$

We compute the limit of each term as $N \rightarrow \infty$ and $\alpha \downarrow 0$. For the first term, applying the residue theorem, we have

$$
\begin{array}{r}
\lim _{N \rightarrow \infty} \sum_{k=1}^{4} I_{4 k}(\alpha, N)=\lim _{N \rightarrow \infty} \frac{1}{(2 \pi i)^{2}} \int_{C_{4}\left(\alpha, N, \omega_{1}\right)} \int_{\partial D_{\alpha, N}\left(\omega_{2}\right)} h\left(t_{1}+t_{2}\right) t_{1}^{m_{1}-1} t_{2}^{m_{2}-1} \\
\times \operatorname{coth}\left(\frac{\pi t_{1}}{\omega_{1}}\right) \operatorname{coth}\left(\frac{\pi t_{2}}{\omega_{2}}\right) d t_{2} d t_{1} \\
=-\frac{\left(\omega_{2} i\right)^{m_{2}}}{2 \pi^{2}} \int_{C_{4}\left(\alpha, N, \omega_{1}\right)}\left(\sum_{n_{2}=1}^{\infty} n_{2}^{m_{2}-1} h\left(t_{1}+n_{2} \omega_{2} i\right)\right) t_{1}^{m_{1}-1} \operatorname{coth}\left(\frac{\pi t_{1}}{\omega_{1}}\right) d t_{1} .
\end{array}
$$

Putting $t_{1}=\alpha e^{i \theta}$ and taking the limit as $\alpha \downarrow 0$, we obtain

$$
\begin{aligned}
\lim _{\alpha \downarrow 0} \lim _{N \rightarrow \infty} & \sum_{k=1}^{4} I_{4 k}(\alpha, N) \\
& =-\frac{i\left(\omega_{2} i\right)^{m_{2}}}{2 \pi^{2}} \int_{\pi}^{0} d \theta\left(\sum_{n_{2}=1}^{\infty} n_{2}^{m_{2}-1} H\left(n_{2} \omega_{2}\right)\right) \times \begin{cases}\omega_{1} / \pi & \text { if } m_{1}=1 \\
0 & \text { otherwise }\end{cases} \\
& =\left.\frac{\omega_{1}^{m_{1}} \omega_{2}^{m_{2}} i^{m}}{2 \pi^{2}} \sum_{n_{2}=1}^{\infty} n_{1}^{m_{1}-1} n_{2}^{m_{2}-1} H\left(n_{1} \omega_{1}+n_{2} \omega_{2}\right)\right|_{n_{1}=0} .
\end{aligned}
$$

Similarly the second term tends to

$\lim _{\alpha \downarrow 0} \lim _{N \rightarrow \infty} \sum_{j=1}^{4} I_{j 4}(\alpha, N)=\left.\frac{\omega_{1}^{m_{1}} \omega_{2}^{m_{2}} i^{m}}{2 \pi^{2}} \sum_{n_{1}=1}^{\infty} n_{1}^{m_{1}-1} n_{2}^{m_{2}-1} H\left(n_{1} \omega_{1}+n_{2} \omega_{2}\right)\right|_{n_{2}=0}$. 
For the third term, putting $t_{1}=\alpha e^{i \theta_{1}}$ and $t_{2}=\alpha e^{i \theta_{2}}$ and taking the limit as $\alpha \downarrow 0$, we obtain

$$
\lim _{\alpha \downarrow 0} \lim _{N \rightarrow \infty} I_{44}(\alpha, N)=-\left.\frac{\omega_{1}^{m_{1}} \omega_{2}^{m_{2}} i^{m}}{4 \pi^{2}} n_{1}^{m_{1}-1} n_{2}^{m_{2}-1} H\left(n_{1} \omega_{1}+n_{2} \omega_{2}\right)\right|_{n_{1}=n_{2}=0} .
$$

Therefore we get

$$
\begin{aligned}
& \lim _{\alpha \downarrow 0} \lim _{N \rightarrow \infty} J_{2}(\alpha, N) \\
& \quad=\frac{\omega_{1}^{m_{1}} \omega_{2}^{m_{2}} i^{m}}{\pi^{2}} \sum_{\substack{n_{1}, n_{2} \geq 0 \\
n_{1}=0 \text { or } n_{2}=0}} \varepsilon_{n_{1}, n_{2}} n_{1}^{m_{1}-1} n_{2}^{m_{2}-1} H\left(n_{1} \omega_{1}+n_{2} \omega_{2}\right) .
\end{aligned}
$$

Next we deal with $\lim _{\alpha \downarrow 0} \lim _{N \rightarrow \infty} J_{1}(\alpha, N)$. We have

$$
\lim _{N \rightarrow \infty} J_{1}(\alpha, N)=\sum_{(j, k) \in\{1,3\}^{2}} I_{j k}(\alpha),
$$

where $I_{j k}(\alpha):=(2 \pi i)^{-2} \int_{C_{j}(\alpha)} \int_{C_{k}(\alpha)} \cdots d t_{2} d t_{1}$ in $(2.1)$ and

$$
C_{1}(\alpha):=\{\alpha+i t: t \geq 0\}, \quad C_{3}(\alpha):=\{-\alpha+i t: t \geq 0\} .
$$

We compute that

$$
\begin{aligned}
I_{11}(\alpha)= & \frac{1}{4 \pi^{2}} \int_{0}^{\infty} \int_{0}^{\infty} h\left(2 \alpha+i\left(t_{1}+t_{2}\right)\right)\left(\alpha+i t_{1}\right)^{m_{1}-1}\left(\alpha+i t_{2}\right)^{m_{2}-1} \\
& \times \operatorname{coth}\left(\pi \frac{\alpha+i t_{1}}{\omega_{1}}\right) \operatorname{coth}\left(\pi \frac{\alpha+i t_{2}}{\omega_{2}}\right) d t_{2} d t_{1} \\
= & \frac{1}{4 \pi^{2}} \int_{0}^{\infty} \int_{t_{1}}^{\infty} h(2 \alpha+i t)\left(\alpha+i t_{1}\right)^{m_{1}-1}\left(\alpha+i\left(t-t_{1}\right)\right)^{m_{2}-1} \\
& \times \operatorname{coth}\left(\pi \frac{\alpha+i t_{1}}{\omega_{1}}\right) \operatorname{coth}\left(\pi \frac{\alpha+i\left(t-t_{1}\right)}{\omega_{2}}\right) d t d t_{1} \\
= & \frac{1}{4 \pi^{2}} \int_{0}^{\infty} h(2 \alpha+i t) \int_{0}^{t}\left(\alpha+i t_{1}\right)^{m_{1}-1}\left(\alpha+i\left(t-t_{1}\right)\right)^{m_{2}-1} \\
= & \frac{1}{4 \pi^{2}} \int_{0}^{\infty} h(2 \alpha+i t) K_{1}(t ; \alpha) d t .
\end{aligned}
$$

Similar calculations together with (T3) lead to

$$
I_{33}(\alpha)=\frac{1}{4 \pi^{2}} \int_{-\infty}^{0} h(2 \alpha+i t) K_{1}(t ; \alpha) d t .
$$


Hence we obtain

$$
I_{11}(\alpha)+I_{33}(\alpha)=\frac{1}{4 \pi^{2}} \int_{-\infty}^{\infty} h(2 \alpha+i t) K_{1}(t ; \alpha) d t .
$$

Similarly we have

$$
I_{13}(\alpha)+I_{31}(\alpha)=-\frac{1}{4 \pi^{2}} \int_{-\infty}^{\infty} H(t) K_{2}(t ; \alpha) d t
$$

where

$$
\begin{aligned}
K_{2}(t ; \alpha):=\int_{0}^{t}\left(\alpha+i t_{1}\right)^{m_{1}-1}\left(-\alpha+i\left(t-t_{1}\right)\right)^{m_{2}-1} \\
\quad \times \operatorname{coth}\left(\pi \frac{\alpha+i t_{1}}{\omega_{1}}\right) \operatorname{coth}\left(\pi \frac{-\alpha+i\left(t-t_{1}\right)}{\omega_{2}}\right) d t_{1} .
\end{aligned}
$$

We now calculate and estimate (2.5) and (2.6). Since the case of (2.5) is similar to that of (2.6), we mainly treat (2.5) and omit the detailed calculations and estimates for (2.6).

By the binomial theorem we have

$$
\begin{aligned}
& \text { (2.7) } \quad \int_{-\infty}^{\infty} h(2 \alpha+i t) K_{1}(t ; \alpha) d t \\
& =i^{m-2} \int_{-\infty}^{\infty} h(2 \alpha+i t) \int_{0}^{t} t_{1}^{m_{1}-1}\left(t-t_{1}\right)^{m_{2}-1} \\
& \times \operatorname{coth}\left(\pi \frac{\alpha+i t_{1}}{\omega_{1}}\right) \operatorname{coth}\left(\pi \frac{\alpha+i\left(t-t_{1}\right)}{\omega_{2}}\right) d t_{1} d t
\end{aligned}
$$

$$
+\left[\begin{array}{c}
\alpha^{l_{0}} \int_{-\infty}^{\infty} h(2 \alpha+i t) \int_{0}^{t} t_{1}^{l_{1}}\left(t-t_{1}\right)^{l_{2}} \\
\quad \times \operatorname{coth}\left(\pi \frac{\alpha+i t_{1}}{\omega_{1}}\right) \operatorname{coth}\left(\pi \frac{\alpha+i\left(t-t_{1}\right)}{\omega_{2}}\right) d t_{1} d t \\
\left(1 \leq l_{0} \leq m-2 ; 0 \leq l_{1} \leq m_{1}-1 ; 0 \leq l_{2} \leq m_{2}-1\right)
\end{array}\right],
$$

where the coefficients of the linear combination depend only on $m_{j}, l_{k}(j=$ $1,2 ; k=0,1,2)$. We first calculate the above integrals with respect to $t_{1}$. Since

$$
\operatorname{coth}(\pi z)=\frac{1+e^{-2 \pi z}}{1-e^{-2 \pi z}}=2 \sum_{n=0}^{\infty} \varepsilon_{n} e^{-2 \pi n z} \quad \text { for } \operatorname{Re}(z)>0
$$


we have

$$
\text { 8) } \begin{aligned}
& \int_{0}^{t} t_{1}^{l_{1}}\left(t-t_{1}\right)^{l_{2}} \operatorname{coth}\left(\pi \frac{\alpha+i t_{1}}{\omega_{1}}\right) \operatorname{coth}\left(\pi \frac{\alpha+i\left(t-t_{1}\right)}{\omega_{2}}\right) d t_{1} \\
= & 4 \sum_{n_{1}=0}^{\infty} \sum_{n_{2}=0}^{\infty} \varepsilon_{n_{1}, n_{2}} e^{-2 \pi \alpha\left(\frac{n_{1}}{\omega_{1}}+\frac{n_{2}}{\omega_{2}}\right)} e\left(-\frac{n_{2} t}{\omega_{2}}\right) \int_{0}^{t} t_{1}^{l_{1}}\left(t-t_{1}\right)^{l_{2}} e^{2 \pi i\left(\frac{n_{2}}{\omega_{2}}-\frac{n_{1}}{\omega_{1}}\right) t_{1}} d t_{1} .
\end{aligned}
$$

We now calculate $\int_{0}^{t} t_{1}^{l_{1}}\left(t-t_{1}\right)^{l_{2}} e^{\beta t_{1}} d t_{1}$ for any $\beta \in \mathbb{C}$. When $\beta=0$, we have

$$
\begin{aligned}
\int_{0}^{t} t_{1}^{l_{1}}\left(t-t_{1}\right)^{l_{2}} d t_{1} & =t^{l_{1}+l_{2}+1} \int_{0}^{1} t_{1}^{l_{1}}\left(1-t_{1}\right)^{l_{2}} d t_{1} \\
& =\frac{l_{1} ! l_{2} !}{\left(l_{1}+l_{2}+1\right) !} t^{l_{1}+l_{2}+1} .
\end{aligned}
$$

In the case $\beta \neq 0$, since for any $L \in \mathbb{Z}_{\geq 0}$ we have

$$
\int_{0}^{t} t_{1}^{L} e^{\beta t_{1}} d t_{1}=(-1)^{L} \frac{L !}{\beta^{L+1}}\left(e^{\beta t} \sum_{k=0}^{L} \frac{(-\beta t)^{k}}{k !}-1\right),
$$

which is easily proved by induction with respect to $L$, we compute that

$$
\begin{aligned}
& \int_{0}^{t} t_{1}^{l_{1}}\left(t-t_{1}\right)^{l_{2}} e^{\beta t_{1}} d t_{1} \\
& =\sum_{k=0}^{l_{2}}(-1)^{k}\left(\begin{array}{l}
l_{2} \\
k
\end{array}\right) t^{l_{2}-k} \int_{0}^{t} t_{1}^{l_{1}+k} e^{\beta t_{1}} d t_{1} \\
& =(-1)^{l_{1}}\left(e^{\beta t} \sum_{k=0}^{l_{2}} \sum_{k^{\prime}=0}^{l_{1}+k}(-1)^{k^{\prime}}\left(\begin{array}{c}
l_{2} \\
k
\end{array}\right) \frac{\left(l_{1}+k\right) !}{k^{\prime} !} \frac{t^{l_{2}-k+k^{\prime}}}{\beta^{l_{1}+k-k^{\prime}+1}}\right. \\
& =:(-1)^{l_{1}}\left(I I_{1}+\sum_{2}^{l_{2}}\right) .
\end{aligned}
$$

To compute $I I_{1}$, replacing $k^{\prime}$ by $l_{1}+k-k^{\prime}$, we have

$$
\begin{aligned}
I_{1} & =(-1)^{l_{1}} e^{\beta t} \sum_{k=0}^{l_{2}} \sum_{k^{\prime}=0}^{l_{1}+k}(-1)^{k+k^{\prime}}\left(\begin{array}{l}
l_{2} \\
k
\end{array}\right) \frac{\left(l_{1}+k\right) !}{\left(l_{1}+k-k^{\prime}\right) !} \frac{t^{l_{1}+l_{2}-k^{\prime}}}{\beta^{k^{\prime}+1}} \\
& =(-1)^{l_{1}} e^{\beta t} \sum_{k^{\prime}=0}^{l_{1}+l_{2}}(-1)^{k^{\prime}} \frac{t^{l_{1}+l_{2}-k^{\prime}}}{\beta^{k^{\prime}+1}}\left(\sum_{k=\max \left\{k^{\prime}-l_{1}, 0\right\}}^{l_{2}}(-1)^{k}\left(\begin{array}{c}
l_{2} \\
k
\end{array}\right) \frac{\left(l_{1}+k\right) !}{\left(l_{1}+k-k^{\prime}\right) !}\right) .
\end{aligned}
$$


The sum over $k$ is calculated as follows:

$$
\begin{aligned}
\sum_{k=\max \left\{k^{\prime}-l_{1}, 0\right\}}^{l_{2}}(-1)^{k}\left(\begin{array}{l}
l_{2} \\
k
\end{array}\right) \frac{\left(l_{1}+k\right) !}{\left(l_{1}+k-k^{\prime}\right) !} \\
=\left.\frac{d^{k^{\prime}}}{d u^{k^{\prime}}}\left(\sum_{k=\max \left\{k^{\prime}-l_{1}, 0\right\}}^{l_{2}}(-1)^{k}\left(\begin{array}{c}
l_{2} \\
k
\end{array}\right) u^{l_{1}+k}\right)\right|_{u=1} \\
=\left.\frac{d^{k^{\prime}}}{d u^{k^{\prime}}}\left(\sum_{k=0}^{l_{2}}(-1)^{k}\left(\begin{array}{c}
l_{2} \\
k
\end{array}\right) u^{l_{1}+k}\right)\right|_{u=1} \\
=\left.\frac{d^{k^{\prime}}}{d u^{k^{\prime}}}\left(u^{l_{1}}(1-u)^{l_{2}}\right)\right|_{u=1} \\
= \begin{cases}0 & \text { if } k^{\prime}=0, \ldots, l_{2}-1, \\
(-1)^{l_{2}}\left(\begin{array}{l}
k^{\prime} \\
l_{2}
\end{array}\right) \frac{l_{1} ! l_{2} !}{\left(l_{1}+l_{2}-k^{\prime}\right) !} & \text { if } k^{\prime}=l_{2}, \ldots, l_{1}+l_{2} .\end{cases}
\end{aligned}
$$

Hence we obtain

$$
\begin{aligned}
I I_{1} & =(-1)^{l_{1}+l_{2}} e^{\beta t} \sum_{k^{\prime}=l_{2}}^{l_{1}+l_{2}}(-1)^{k^{\prime}} \frac{t^{l_{1}+l_{2}-k^{\prime}}}{\beta^{k^{\prime}+1}}\left(\begin{array}{c}
k^{\prime} \\
l_{2}
\end{array}\right) \frac{l_{1} ! l_{2} !}{\left(l_{1}+l_{2}-k^{\prime}\right) !} \\
& =(-1)^{l_{1}} e^{\beta t} \sum_{k=0}^{l_{1}}(-1)^{k} \frac{t^{l_{1}-k}}{\beta^{l_{2}+k+1}} \frac{\left(l_{2}+k\right) !}{l_{2} ! k !} \frac{l_{1} ! l_{2} !}{\left(l_{1}-k\right) !} \\
& =(-1)^{l_{1}} e^{\beta t} \sum_{k=0}^{l_{1}}(-1)^{k}\left(\begin{array}{c}
l_{1} \\
k
\end{array}\right)\left(l_{2}+k\right) ! \frac{t^{l_{1}-k}}{\beta^{l_{2}+k+1}} .
\end{aligned}
$$

Therefore,

$$
\begin{aligned}
& \int_{0}^{t} t_{1}^{l_{1}}\left(t-t_{1}\right)^{l_{2}} e^{\beta t_{1}} d t_{1} \\
& =e^{\beta t} \sum_{k=0}^{l_{1}}(-1)^{k}\left(\begin{array}{l}
l_{1} \\
k
\end{array}\right)\left(l_{2}+k\right) ! \frac{t^{l_{1}-k}}{\beta^{l_{2}+k+1}} \\
& +(-1)^{l_{1}+1} \sum_{k=0}^{l_{2}}\left(\begin{array}{l}
l_{2} \\
k
\end{array}\right)\left(l_{1}+k\right) ! \frac{t^{l_{2}-k}}{\beta^{l_{1}+k+1}} .
\end{aligned}
$$

We go back to the calculation of (2.8). Since $\omega_{1} / \omega_{2}$ is generic, for any $n_{1}, n_{2} \in \mathbb{Z}_{\geq 0}$ we have $n_{1} \omega_{1}-n_{2} \omega_{2}=0$ if and only if $\left(n_{1}, n_{2}\right)=(0,0)$. 
Applying (2.9) and (2.10) to (2.8), we get

$$
\begin{aligned}
& \int_{0}^{t} t_{1}^{l_{1}}\left(t-t_{1}\right)^{l_{2}} \operatorname{coth}\left(\pi \frac{\alpha+i t_{1}}{\omega_{1}}\right) \operatorname{coth}\left(\pi \frac{\alpha+i\left(t-t_{1}\right)}{\omega_{2}}\right) d t_{1} \\
& =\frac{l_{1} ! l_{2} !}{\left(l_{1}+l_{2}+1\right) !} t^{l_{1}+l_{2}+1} \\
& +4(-1)^{l_{2}+1} \sum_{k=0}^{l_{1}}\left(\begin{array}{l}
l_{1} \\
k
\end{array}\right) \frac{\left(l_{2}+k\right) !}{(2 \pi i)^{l_{2}+k+1}} \\
& \times \sum_{\substack{n_{1}, n_{2} \geq 0 \\
\left(n_{1}, n_{2}\right) \neq(0,0)}} \varepsilon_{n_{1}, n_{2}} \frac{e^{-2 \pi \alpha\left(\frac{n_{1}}{\omega_{1}}+\frac{n_{2}}{\omega_{2}}\right)}}{\left(\frac{n_{1}}{\omega_{1}}-\frac{n_{2}}{\omega_{2}}\right)^{l_{2}+k+1}} t^{l_{1}-k} e\left(-\frac{n_{1}}{\omega_{1}} t\right) \\
& +4(-1)^{l_{1}+1} \sum_{k=0}^{l_{2}}\left(\begin{array}{c}
l_{2} \\
k
\end{array}\right) \frac{\left(l_{1}+k\right) !}{(2 \pi i)^{l_{1}+k+1}} \\
& \times \sum_{\substack{n_{1}, n_{2} \geq 0 \\
\left(n_{1}, n_{2}\right) \neq(0,0)}} \varepsilon_{n_{1}, n_{2}} \frac{e^{-2 \pi \alpha\left(\frac{n_{1}}{\omega_{1}}+\frac{n_{2}}{\omega_{2}}\right)}}{\left(\frac{n_{2}}{\omega_{2}}-\frac{n_{1}}{\omega_{1}}\right)^{l_{1}+k+1}} t^{l_{2}-k} e\left(-\frac{n_{2}}{\omega_{2}} t\right) \\
& =: I I I_{1}(t)+I I I_{2}(t)+I I I_{3}(t) .
\end{aligned}
$$

Lemma 6.5 guarantees the absolute convergence of the sums in (2.11). We now calculate and estimate the integrals of (2.7) with respect to $t_{1}$ and $t$ as $\alpha \downarrow 0$. For this purpose we compute $\int_{-\infty}^{\infty} h(2 \alpha+i t) I I I_{j}(t) d t$. From Lemma 6.1 we obtain

$$
\begin{aligned}
\lim _{\alpha \downarrow 0} \int_{-\infty}^{\infty} h(2 \alpha+i t) I I I_{1}(t) & d t \\
& =\frac{1}{(-2 \pi i)^{l_{1}+l_{2}+1}} \frac{l_{1} ! l_{2} !}{\left(l_{1}+l_{2}+1\right) !} \widehat{H}^{\left(l_{1}+l_{2}+1\right)}(0) .
\end{aligned}
$$

Next, we consider $\int_{-\infty}^{\infty} h(2 \alpha+i t) I I I_{2}(t) d t$. It follows from Lemmas 6.3 and 6.6 that for any $k=0, \ldots, l_{1}$ we have

$$
\begin{aligned}
\int_{-\infty}^{\infty} h(2 \alpha+i t) & \sum_{\substack{n_{1}, n_{2} \geq 0 \\
\left(n_{1}, n_{2}\right) \neq(0,0)}} \varepsilon_{n_{1}, n_{2}} \frac{e^{-2 \pi \alpha\left(\frac{n_{1}}{\omega_{1}}+\frac{n_{2}}{\omega_{2}}\right)}}{\left(\frac{n_{1}}{\omega_{1}}-\frac{n_{2}}{\omega_{2}}\right)^{l_{2}+k+1}} t^{l_{1}-k} e\left(-\frac{n_{1}}{\omega_{1}} t\right) d t \\
= & \sum_{\substack{n_{1}, n_{2} \geq 0 \\
\left(n_{1}, n_{2}\right) \neq(0,0)}} \varepsilon_{n_{1}, n_{2}} \frac{e^{-2 \pi \alpha\left(\frac{n_{1}}{\omega_{1}}+\frac{n_{2}}{\omega_{2}}\right)}}{\left(\frac{n_{1}}{\omega_{1}}-\frac{n_{2}}{\omega_{2}}\right)^{l_{2}+k+1}} \int_{-\infty}^{\infty} t^{l_{1}-k} h(2 \alpha+i t) e\left(-\frac{n_{1}}{\omega_{1}} t\right) d t
\end{aligned}
$$




$$
\begin{aligned}
& =\frac{1}{(-2 \pi i)^{l_{1}-k}} \sum_{\substack{n_{1}, n_{2} \geq 0 \\
\left(n_{1}, n_{2}\right) \neq(0,0)}} \varepsilon_{n_{1}, n_{2}} \frac{e^{2 \pi \alpha\left(\frac{n_{1}}{\omega_{1}}-\frac{n_{2}}{\omega_{2}}\right)}}{\left(\frac{n_{1}}{\omega_{1}}-\frac{n_{2}}{\omega_{2}}\right)^{l_{2}+k+1}} \widehat{H}^{\left(l_{1}-k\right)}\left(\frac{n_{1}}{\omega_{1}}\right) \\
& +O\left(\alpha \sum_{\substack{n_{1}, n_{2} \geq 0 \\
\left(n_{1}, n_{2}\right) \neq(0,0)}} \frac{e^{-2 \pi \alpha\left(\frac{n_{1}}{\omega_{1}}+\frac{n_{2}}{\omega_{2}}\right)}}{\left|\frac{n_{1}}{\omega_{1}}-\frac{n_{2}}{\omega_{2}}\right|^{l_{2}+k+1}} e^{-\pi A \frac{n_{1}}{\omega_{1}}}\right) \\
& =\frac{1}{(-2 \pi i)^{l_{1}-k}} \sum_{\substack{n_{1}, n_{2} \geq 0 \\
\left(n_{1}, n_{2}\right) \neq(0,0)}} \varepsilon_{n_{1}, n_{2}} \frac{e^{2 \pi \alpha\left(\frac{n_{1}}{\omega_{1}}-\frac{n_{2}}{\omega_{2}}\right)}}{\left(\frac{n_{1}}{\omega_{1}}-\frac{n_{2}}{\omega_{2}}\right)^{l_{2}+k+1}} \widehat{H}^{\left(l_{1}-k\right)}\left(\frac{n_{1}}{\omega_{1}}\right) \\
& +O(\alpha \log (1 / \alpha))
\end{aligned}
$$

as $\alpha \downarrow 0$. Hence as $\alpha \downarrow 0$, we have

$$
\begin{aligned}
\int_{-\infty}^{\infty} h(2 \alpha+i t) I I I_{2}(t) d t & \\
= & \frac{4}{(-2 \pi i)^{l_{1}+l_{2}+1}} \sum_{k=0}^{l_{1}}(-1)^{k}\left(\begin{array}{c}
l_{1} \\
k
\end{array}\right)\left(l_{2}+k\right) ! \\
& \times \sum_{\substack{n_{1}, n_{2} \geq 0 \\
\left(n_{1}, n_{2}\right) \neq(0,0)}} \varepsilon_{n_{1}, n_{2}} \frac{e^{2 \pi \alpha\left(\frac{n_{1}}{\omega_{1}}-\frac{n_{2}}{\omega_{2}}\right)}}{\left(\frac{n_{1}}{\omega_{1}}-\frac{n_{2}}{\omega_{2}}\right)^{l_{2}+k+1}} \widehat{H}^{\left(l_{1}-k\right)}\left(\frac{n_{1}}{\omega_{1}}\right)+o(1) .
\end{aligned}
$$

In particular, estimating the first term by Lemmas 6.2 and 6.6 , we obtain

$$
\int_{-\infty}^{\infty} h(2 \alpha+i t) I I I_{2}(t) d t=O(\log (1 / \alpha)) \quad \text { as } \alpha \downarrow 0 .
$$

Similar calculations and estimates give

$$
\begin{aligned}
\int_{-\infty}^{\infty} h(2 \alpha+i t) I I I_{3}(t) d t & \\
= & \frac{4}{(-2 \pi i)^{l_{1}+l_{2}+1}} \sum_{k=0}^{l_{2}}(-1)^{k}\left(\begin{array}{c}
l_{2} \\
k
\end{array}\right)\left(l_{1}+k\right) ! \\
& \times \sum_{\substack{n_{1}, n_{2} \geq 0 \\
\left(n_{1}, n_{2}\right) \neq(0,0)}} \varepsilon_{n_{1}, n_{2}} \frac{e^{2 \pi \alpha\left(\frac{n_{2}}{\omega_{2}}-\frac{n_{1}}{\omega_{1}}\right)}}{\left(\frac{n_{2}}{\omega_{2}}-\frac{n_{1}}{\omega_{1}}\right)^{l_{1}+k+1}} \widehat{H}^{\left(l_{2}-k\right)}\left(\frac{n_{2}}{\omega_{2}}\right)+o(1)
\end{aligned}
$$

and 


$$
\int_{-\infty}^{\infty} h(2 \alpha+i t) I I I_{3}(t) d t=O(\log (1 / \alpha))
$$

as $\alpha \downarrow 0$. Therefore, it follows from (2.11)-(2.16) that

$$
\begin{aligned}
& \text { (2.17) } \int_{-\infty}^{\infty} h(2 \alpha+i t) \int_{0}^{t} t_{1}^{l_{1}}\left(t-t_{1}\right)^{l_{2}} \\
& \quad \times \operatorname{coth}\left(\pi \frac{\alpha+i t_{1}}{\omega_{1}}\right) \operatorname{coth}\left(\pi \frac{\alpha+i\left(t-t_{1}\right)}{\omega_{2}}\right) d t_{1} d t \\
& =\frac{1}{(-2 \pi i)^{l_{1}+l_{2}+1}}\left(\frac{l_{1} ! l_{2} !}{\left(l_{1}+l_{2}+1\right) !} \widehat{H}^{\left(l_{1}+l_{2}+1\right)}(0)\right.
\end{aligned}
$$$$
+4 \sum_{k=0}^{l_{1}}(-1)^{k}\left(\begin{array}{l}
l_{1} \\
k
\end{array}\right)\left(l_{2}+k\right) ! \sum_{\substack{n_{1}, n_{2} \geq 0 \\
\left(n_{1}, n_{2}\right) \neq(0,0)}} \varepsilon_{n_{1}, n_{2}} \frac{e^{2 \pi \alpha\left(\frac{n_{1}}{\omega_{1}}-\frac{n_{2}}{\omega_{2}}\right)}}{\left(\frac{n_{1}}{\omega_{1}}-\frac{n_{2}}{\omega_{2}}\right)^{l_{2}+k+1}} \widehat{H}^{\left(l_{1}-k\right)}\left(\frac{n_{1}}{\omega_{1}}\right)
$$$$
\left.+4 \sum_{k=0}^{l_{2}}(-1)^{k}\left(\begin{array}{c}
l_{2} \\
k
\end{array}\right)\left(l_{1}+k\right) ! \sum_{\substack{n_{1}, n_{2} \geq 0 \\
\left(n_{1}, n_{2}\right) \neq(0,0)}} \varepsilon_{n_{1}, n_{2}} \frac{e^{2 \pi \alpha\left(\frac{n_{2}}{\omega_{2}}-\frac{n_{1}}{\omega_{1}}\right)}}{\left(\frac{n_{2}}{\omega_{2}}-\frac{n_{1}}{\omega_{1}}\right)^{l_{1}+k+1}} \widehat{H}^{\left(l_{2}-k\right)}\left(\frac{n_{2}}{\omega_{2}}\right)\right)
$$$$
+o(1)
$$

and

$$
\begin{aligned}
& \int_{-\infty}^{\infty} h(2 \alpha+i t) \int_{0}^{t} t_{1}^{l_{1}}\left(t-t_{1}\right)^{l_{2}} \\
& \quad \times \operatorname{coth}\left(\pi \frac{\alpha+i t_{1}}{\omega_{1}}\right) \operatorname{coth}\left(\pi \frac{\alpha+i\left(t-t_{1}\right)}{\omega_{2}}\right) d t_{1} d t=O(\log (1 / \alpha))
\end{aligned}
$$

as $\alpha \downarrow 0$. Applying (2.17) to the first term of (2.7), and (2.18) to the second, gives

$$
\begin{aligned}
\int_{-\infty}^{\infty} h(2 \alpha+i t) & K_{1}(t ; \alpha) d t \\
= & \frac{1}{(-2 \pi)^{m-1} i}\left(\frac{\left(m_{1}-1\right) !\left(m_{2}-1\right) !}{(m-1) !} \widehat{H}^{(m-1)}(0)\right. \\
& +4 \sum_{k=0}^{m_{1}-1}(-1)^{k}\left(\begin{array}{c}
m_{1}-1 \\
k
\end{array}\right)\left(m_{2}+k-1\right) ! \\
& \times \sum_{\substack{n_{1}, n_{2} \geq 0 \\
\left(n_{1}, n_{2}\right) \neq(0,0)}} \varepsilon_{n_{1}, n_{2}} \frac{e^{2 \pi \alpha\left(\frac{n_{1}}{\omega_{1}}-\frac{n_{2}}{\omega_{2}}\right)}}{\left(\frac{n_{1}}{\omega_{1}}-\frac{n_{2}}{\omega_{2}}\right)^{m_{2}+k}} \widehat{H}^{\left(m_{1}-k-1\right)}\left(\frac{n_{1}}{\omega_{1}}\right)
\end{aligned}
$$




$$
\begin{aligned}
& +4 \sum_{k=0}^{m_{2}-1}(-1)^{k}\left(\begin{array}{c}
m_{2}-1 \\
k
\end{array}\right)\left(m_{1}+k-1\right) ! \\
& \left.\times \sum_{\substack{n_{1}, n_{2} \geq 0 \\
\left(n_{1}, n_{2}\right) \neq(0,0)}} \varepsilon_{n_{1}, n_{2}} \frac{e^{2 \pi \alpha\left(\frac{n_{2}}{\omega_{2}}-\frac{n_{1}}{\omega_{1}}\right)}}{\left(\frac{n_{2}}{\omega_{2}}-\frac{n_{1}}{\omega_{1}}\right)^{m_{1}+k}} \widehat{H}^{\left(m_{2}-k-1\right)}\left(\frac{n_{2}}{\omega_{2}}\right)\right)+o(1) \quad \text { as } \alpha \downarrow 0 .
\end{aligned}
$$

We calculate the integral $\int_{-\infty}^{\infty} H(t) K_{2}(t ; \alpha) d t$ in the same manner as $\int_{-\infty}^{\infty} h(2 \alpha+i t) K_{1}(t ; \alpha) d t$. Since the calculations do not need more efforts, we omit the details. The formula corresponding to (2.19) is

$$
\begin{aligned}
& \text { (2.20) } \quad \int_{-\infty}^{\infty} H(t) K_{2}(t ; \alpha) d t \\
& =-\frac{1}{(-2 \pi)^{m-1} i}\left(\frac{\left(m_{1}-1\right) !\left(m_{2}-1\right) !}{(m-1) !} \widehat{H}^{(m-1)}(0)\right.
\end{aligned}
$$

$$
+4 \sum_{k=0}^{m_{1}-1}(-1)^{k}\left(\begin{array}{c}
m_{1}-1 \\
k
\end{array}\right)\left(m_{2}+k-1\right) \text { ! }
$$$$
\times \sum_{\substack{n_{1}, n_{2} \geq 0 \\\left(n_{1}, n_{2}\right) \neq(0,0)}} \varepsilon_{n_{1}, n_{2}} \frac{e^{-2 \pi \alpha\left(\frac{n_{1}}{\omega_{1}}+\frac{n_{2}}{\omega_{2}}\right)}}{\left(\frac{n_{1}}{\omega_{1}}+\frac{n_{2}}{\omega_{2}}\right)^{m_{2}+k}} \widehat{H}^{\left(m_{1}-k-1\right)}\left(\frac{n_{1}}{\omega_{1}}\right)
$$

$$
\begin{aligned}
& +4 \sum_{k=0}^{m_{2}-1}(-1)^{k}\left(\begin{array}{c}
m_{2}-1 \\
k
\end{array}\right)\left(m_{1}+k-1\right) ! \\
& \left.\times \sum_{\substack{n_{1}, n_{2} \geq 0 \\
\left(n_{1}, n_{2}\right) \neq(0,0)}} \varepsilon_{n_{1}, n_{2}} \frac{e^{-2 \pi \alpha\left(\frac{n_{2}}{\omega_{2}}+\frac{n_{1}}{\omega_{1}}\right)}}{\left(\frac{n_{2}}{\omega_{2}}+\frac{n_{1}}{\omega_{1}}\right)^{m_{1}+k}} \widehat{H}^{\left(m_{2}-k-1\right)}\left(\frac{n_{2}}{\omega_{2}}\right)\right)+o(1) \quad \text { as } \alpha \downarrow 0 .
\end{aligned}
$$

We go back to (2.4)-(2.6). By (2.19) and (2.20) we have

$$
\begin{aligned}
\lim _{N \rightarrow \infty} J_{1}(\alpha, N)= & \frac{1}{\pi^{2}} \frac{1}{(-2 \pi)^{m-1} i}\left(\frac{1}{2} \frac{\left(m_{1}-1\right) !\left(m_{2}-1\right) !}{(m-1) !} \widehat{H}^{(m-1)}(0)\right. \\
& +\sum_{k=0}^{m_{1}-1}(-1)^{k}\left(\begin{array}{c}
m_{1}-1 \\
k
\end{array}\right)\left(m_{2}+k-1\right) ! I V_{1}(\alpha ; k) \\
& \left.+\sum_{k=0}^{m_{2}-1}(-1)^{k}\left(\begin{array}{c}
m_{2}-1 \\
k
\end{array}\right)\left(m_{1}+k-1\right) ! I V_{2}(\alpha ; k)\right) \\
& +o(1) \text { as } \alpha \downarrow 0,
\end{aligned}
$$


where

$$
\begin{aligned}
& I V_{1}(\alpha ; k) \\
& :=\sum_{\substack{n_{1}, n_{2} \geq 0 \\
\left(n_{1}, n_{2}\right) \neq(0,0)}} \varepsilon_{n_{1}, n_{2}}\left(\frac{e^{2 \pi \alpha\left(\frac{n_{1}}{\omega_{1}}-\frac{n_{2}}{\omega_{2}}\right)}}{\left(\frac{n_{1}}{\omega_{1}}-\frac{n_{2}}{\omega_{2}}\right)^{m_{2}+k}}+\frac{e^{-2 \pi \alpha\left(\frac{n_{1}}{\omega_{1}}+\frac{n_{2}}{\omega_{2}}\right)}}{\left(\frac{n_{1}}{\omega_{1}}+\frac{n_{2}}{\omega_{2}}\right)^{m_{2}+k}}\right) \widehat{H}^{\left(m_{1}-k-1\right)}\left(\frac{n_{1}}{\omega_{1}}\right), \\
& I V_{2}(\alpha ; k) \\
& :=\sum_{\substack{n_{1}, n_{2} \geq 0 \\
\left(n_{1}, n_{2}\right) \neq(0,0)}} \varepsilon_{n_{1}, n_{2}}\left(\frac{e^{2 \pi \alpha\left(\frac{n_{2}}{\omega_{2}}-\frac{n_{1}}{\omega_{1}}\right)}}{\left(\frac{n_{2}}{\omega_{2}}-\frac{n_{1}}{\omega_{1}}\right)^{m_{1}+k}}+\frac{e^{-2 \pi \alpha\left(\frac{n_{2}}{\omega_{2}}+\frac{n_{1}}{\omega_{1}}\right)}}{\left(\frac{n_{2}}{\omega_{2}}+\frac{n_{1}}{\omega_{1}}\right)^{m_{1}+k}}\right) \widehat{H}^{\left(m_{2}-k-1\right)}\left(\frac{n_{2}}{\omega_{2}}\right) .
\end{aligned}
$$

As $\alpha \downarrow 0$, we obtain

$$
\begin{aligned}
= & \frac{1}{\pi^{2}} \frac{1}{(-2 \pi)^{m-1} i}\left(\frac{1}{2} \frac{\left(m_{1}-1\right) !\left(m_{2}-1\right) !}{(m-1) !} \widehat{H}^{(m-1)}(0)\right. \\
& +\sum_{k=0}^{m_{1}-1}(-1)^{k}\left(\begin{array}{c}
m_{1}-1 \\
k
\end{array}\right)\left(m_{2}+k-1\right) ! \\
& \times \sum_{\substack{n_{1}, n_{2} \geq 0 \\
\left(n_{1}, n_{2}\right) \neq(0,0)}} \varepsilon_{n_{1}, n_{2}}\left(\frac{1}{\left(\frac{n_{1}}{\omega_{1}}-\frac{n_{2}}{\omega_{2}}\right)^{m_{2}+k}}+\frac{1}{\left(\frac{n_{1}}{\omega_{1}}+\frac{n_{2}}{\omega_{2}}\right)^{m_{2}+k}}\right) \widehat{H}^{\left(m_{1}-k-1\right)}\left(\frac{n_{1}}{\omega_{1}}\right) \\
& +\sum_{\substack{k=0 \\
m_{2}-1}}(-1)^{k}\left(\begin{array}{c}
m_{2}-1 \\
k
\end{array}\right)\left(m_{1}+k-1\right) ! \\
& \left.\times \sum_{\substack{n_{1}, n_{2} \geq 0 \\
\left(n_{1}, n_{2}\right) \neq(0,0)}} \varepsilon_{n_{1}, n_{2}}\left(\frac{1}{\left(\frac{n_{2}}{\omega_{2}}-\frac{n_{1}}{\omega_{1}}\right)^{m_{1}+k}}+\frac{1}{\left(\frac{n_{2}}{\omega_{2}}+\frac{n_{1}}{\omega_{1}}\right)^{m_{1}+k}}\right) \widehat{H}^{\left(m_{2}-k-1\right)}\left(\frac{n_{2}}{\omega_{2}}\right)\right) .
\end{aligned}
$$

(2.2), (2.3) and (2.22) complete the proof.

3. Multiple sine functions. In this section we prove Theorem 2 . Throughout this section, $M, N, r, \omega_{1}, \omega_{2}, \underline{\omega}$ are as in Theorem 2.

Proof of Theorem 2. We put

$$
\varphi_{r}(s, z, \underline{\omega}):=-\zeta_{r}(s, z, \underline{\omega})+(-1)^{r} \zeta_{r}(s,|\underline{\omega}|-z, \underline{\omega}) .
$$

Then

$$
S_{r}(z, \underline{\omega})=\exp \left(\left.\frac{\partial}{\partial s} \varphi_{r}(s, z, \underline{\omega})\right|_{s=0}\right) .
$$


We calculate $\zeta_{r}(s, z, \underline{\omega})$ for $\operatorname{Re}(s)>r$ :

$$
\begin{aligned}
\zeta_{r}(s, z, \underline{\omega}) & =\sum_{n_{1}, \ldots, n_{r} \geq 0}\left(\left(n_{1}+\cdots+n_{M}\right) \omega_{1}+\left(n_{M+1}+\cdots+n_{r}\right) \omega_{2}+z\right)^{-s} \\
& =\sum_{n_{1}, n_{2} \geq 0}\left(\begin{array}{c}
n_{1}+M-1 \\
M-1
\end{array}\right)\left(\begin{array}{c}
n_{2}+N-1 \\
N-1
\end{array}\right)\left(n_{1} \omega_{1}+n_{2} \omega_{2}+z\right)^{-s} \\
& =(-1)^{r} \sum_{n_{1}, n_{2} \geq 0} g_{M}\left(-n_{1}\right) g_{N}\left(-n_{2}\right)\left(n_{1} \omega_{1}+n_{2} \omega_{2}+z\right)^{-s} \\
& =(-1)^{r} \sum_{m_{1}=0}^{M-1} \sum_{m_{2}=0}^{N-1}(-1)^{m_{1}+m_{2}} a_{M, m_{1}} a_{N, m_{2}} \zeta_{\left(m_{1}+1, m_{2}+1\right)}^{1}\left(s, z,\left(\omega_{1}, \omega_{2}\right)\right)
\end{aligned}
$$

where $g_{M}$ is defined as in (1.1) and

$$
\begin{gathered}
g_{M}(x)=: \sum_{m=0}^{M-1} a_{M, m} x^{m} \\
\zeta_{\left(m_{1}, m_{2}\right)}^{1}\left(s, z,\left(\omega_{1}, \omega_{2}\right)\right):=\sum_{n_{1}, n_{2} \geq 0} \frac{n_{1}^{m_{1}-1} n_{2}^{m_{2}-1}}{\left(n_{1} \omega_{1}+n_{2} \omega_{2}+z\right)^{s}} .
\end{gathered}
$$

Similar calculations together with $g_{M}(1)=\cdots=g_{M}(M-1)=0$ give

$$
\zeta_{r}(s,|\underline{\omega}|-z, \underline{\omega})=\sum_{m_{1}=0}^{M-1} \sum_{m_{2}=0}^{N-1} a_{M, m_{1}} a_{N, m_{2}} \zeta_{\left(m_{1}+1, m_{2}+1\right)}^{2}\left(s, z,\left(\omega_{1}, \omega_{2}\right)\right),
$$

where

$$
\zeta_{\left(m_{1}, m_{2}\right)}^{2}\left(s, z,\left(\omega_{1}, \omega_{2}\right)\right):=\sum_{n_{1}, n_{2} \geq 1} \frac{n_{1}^{m_{1}-1} n_{2}^{m_{2}-1}}{\left(n_{1} \omega_{1}+n_{2} \omega_{2}-z\right)^{s}}
$$

Hence we obtain

$$
\begin{aligned}
& \varphi_{r}(s, z, \underline{\omega}) \\
& \quad=(-1)^{r} \sum_{m_{1}=0}^{M-1} \sum_{m_{2}=0}^{N-1} a_{M, m_{1}} a_{N, m_{2}} \Phi_{\left(m_{1}+1, m_{2}+1\right)}\left(s, z,\left(\omega_{1}, \omega_{2}\right)\right),
\end{aligned}
$$

where

$$
\begin{aligned}
\Phi_{\left(m_{1}, m_{2}\right)}\left(s, z,\left(\omega_{1}, \omega_{2}\right)\right):= & (-1)^{m_{1}+m_{2}+1} \zeta_{\left(m_{1}, m_{2}\right)}^{1}\left(s, z,\left(\omega_{1}, \omega_{2}\right)\right) \\
& +\zeta_{\left(m_{1}, m_{2}\right)}^{2}\left(s, z,\left(\omega_{1}, \omega_{2}\right)\right) .
\end{aligned}
$$


We remark that for fixed $0<\operatorname{Re}(z)<\omega_{1}+\omega_{2}, \zeta_{\left(m_{1}, m_{2}\right)}^{j}\left(s, z,\left(\omega_{1}, \omega_{2}\right)\right)$ admits a meromorphic continuation to $s \in \mathbb{C}$ by the usual method:

$$
\begin{gathered}
\zeta_{\left(m_{1}, m_{2}\right)}^{1}\left(s, z,\left(\omega_{1}, \omega_{2}\right)\right)=-\frac{\Gamma(1-s)}{2 \pi i} \frac{(-1)^{m_{1}+m_{2}}}{\omega_{1}^{m_{1}-1} \omega_{2}^{m_{2}-1}} \\
\times \int_{C_{\varepsilon}} e^{-z u} \frac{d^{m_{1}-1}}{d u^{m_{1}-1}}\left(\frac{1}{1-e^{-\omega_{1} u}}\right) \frac{d^{m_{2}-1}}{d u^{m_{2}-1}}\left(\frac{1}{1-e^{-\omega_{2} u}}\right)(-u)^{s-1} d u \\
\zeta_{\left(m_{1}, m_{2}\right)}^{2}\left(s, z,\left(\omega_{1}, \omega_{2}\right)\right)=-\frac{\Gamma(1-s)}{2 \pi i} \frac{(-1)^{m_{1}+m_{2}}}{\omega_{1}^{m_{1}-1} \omega_{2}^{m_{2}-1}} \\
\times \int_{C_{\varepsilon}} e^{z u} \frac{d^{m_{1}-1}}{d u^{m_{1}-1}}\left(\frac{1}{e^{\omega_{1} u}-1}\right) \frac{d^{m_{2}-1}}{d u^{m_{2}-1}}\left(\frac{1}{e^{\omega_{2} u}-1}\right)(-u)^{s-1} d u
\end{gathered}
$$

where $0<\varepsilon<\min \left\{2 \pi / \omega_{1}, 2 \pi / \omega_{2}\right\}$ is a fixed number and $C_{\varepsilon}$ is the union of $+\infty \rightarrow+\varepsilon, \varepsilon e^{i \theta}(0 \leq \theta \leq 2 \pi)$ and $+\varepsilon \rightarrow+\infty$. Hence (3.2) holds for any $s \in \mathbb{C}$. In particular, we have

$$
\begin{aligned}
& \left.\frac{\partial}{\partial s} \varphi_{r}(s, z, \underline{\omega})\right|_{s=0} \\
= & \left.(-1)^{r} \sum_{m_{1}=0}^{M-1} \sum_{m_{2}=0}^{N-1} a_{M, m_{1}} a_{N, m_{2}} \frac{\partial}{\partial s} \Phi_{\left(m_{1}+1, m_{2}+1\right)}\left(s, z,\left(\omega_{1}, \omega_{2}\right)\right)\right|_{s=0} .
\end{aligned}
$$

We calculate $\left.\frac{\partial}{\partial s} \Phi_{\left(m_{1}, m_{2}\right)}\left(s, z,\left(\omega_{1}, \omega_{2}\right)\right)\right|_{s=0}$ for $m_{1}, m_{2} \in \mathbb{Z}_{\geq 1}$. We have

$$
\begin{aligned}
& \frac{\partial^{m+1}}{\partial z^{m+1}} \zeta_{\left(m_{1}, m_{2}\right)}^{1}\left(s, z,\left(\omega_{1}, \omega_{2}\right)\right) \\
& \quad=(-1)^{m+1} s(s+1) \cdots(s+m) \sum_{n_{1}, n_{2} \geq 0} \frac{n_{1}^{m_{1}-1} n_{2}^{m_{2}-1}}{\left(n_{1} \omega_{1}+n_{2} \omega_{2}+z\right)^{s+m+1}},
\end{aligned}
$$

where $m:=m_{1}+m_{2}$. The series converges absolutely for $\operatorname{Re}(s)>-1$. Hence

$$
\begin{aligned}
& \left.\frac{\partial^{m+2}}{\partial s \partial z^{m+1}} \zeta_{\left(m_{1}, m_{2}\right)}^{1}\left(s, z,\left(\omega_{1}, \omega_{2}\right)\right)\right|_{s=0} \\
& =(-1)^{m+1} m ! \sum_{n_{1}, n_{2} \geq 0} \frac{n_{1}^{m_{1}-1} n_{2}^{m_{2}-1}}{\left(n_{1} \omega_{1}+n_{2} \omega_{2}+z\right)^{m+1}}
\end{aligned}
$$

Similar calculations give

$$
\left.\frac{\partial^{m+2}}{\partial s \partial z^{m+1}} \zeta_{\left(m_{1}, m_{2}\right)}^{2}\left(s, z,\left(\omega_{1}, \omega_{2}\right)\right)\right|_{s=0}=m ! \sum_{n_{1}, n_{2} \geq 1} \frac{n_{1}^{m_{1}-1} n_{2}^{m_{2}-1}}{\left(n_{1} \omega_{1}+n_{2} \omega_{2}-z\right)^{m+1}} .
$$


Therefore

$$
\begin{aligned}
& \left.\frac{\partial^{m+2}}{\partial s \partial z^{m+1}} \Phi_{\left(m_{1}, m_{2}\right)}\left(s, z,\left(\omega_{1}, \omega_{2}\right)\right)\right|_{s=0} \\
& =m !\left(\sum_{n_{1}, n_{2} \geq 0} \frac{n_{1}^{m_{1}-1} n_{2}^{m_{2}-1}}{\left(n_{1} \omega_{1}+n_{2} \omega_{2}+z\right)^{m+1}}+\sum_{n_{1}, n_{2} \geq 1} \frac{n_{1}^{m_{1}-1} n_{2}^{m_{2}-1}}{\left(n_{1} \omega_{1}+n_{2} \omega_{2}-z\right)^{m+1}}\right) \\
& =m !\left\{\sum_{n_{1}, n_{2} \geq 0} \varepsilon_{n_{1}, n_{2}}\left(\frac{n_{1}^{m_{1}-1} n_{2}^{m_{2}-1}}{\left(n_{1} \omega_{1}+n_{2} \omega_{2}+z\right)^{m+1}}+\frac{n_{1}^{m_{1}-1} n_{2}^{m_{2}-1}}{\left(n_{1} \omega_{1}+n_{2} \omega_{2}-z\right)^{m+1}}\right)\right. \\
& +\left.\frac{1}{2} \sum_{n_{1} \geq 0} \varepsilon_{n_{1}}\left(\frac{n_{1}^{m_{1}-1} n_{2}^{m_{2}-1}}{\left(n_{1} \omega_{1}+n_{2} \omega_{2}+z\right)^{m+1}}-\frac{n_{1}^{m_{1}-1} n_{2}^{m_{2}-1}}{\left(n_{1} \omega_{1}+n_{2} \omega_{2}-z\right)^{m+1}}\right)\right|_{n_{2}=0} \\
& +\left.\frac{1}{2} \sum_{n_{2} \geq 0} \varepsilon_{n_{2}}\left(\frac{n_{1}^{m_{1}-1} n_{2}^{m_{2}-1}}{\left(n_{1} \omega_{1}+n_{2} \omega_{2}+z\right)^{m+1}}-\frac{n_{1}^{m_{1}-1} n_{2}^{m_{2}-1}}{\left(n_{1} \omega_{1}+n_{2} \omega_{2}-z\right)^{m+1}}\right)\right|_{n_{1}=0} \\
& \left.+\left.\frac{1}{4}\left(\frac{n_{1}^{m_{1}-1} n_{2}^{m_{2}-1}}{\left(n_{1} \omega_{1}+n_{2} \omega_{2}+z\right)^{m+1}}+\frac{n_{1}^{m_{1}-1} n_{2}^{m_{2}-1}}{\left(n_{1} \omega_{1}+n_{2} \omega_{2}-z\right)^{m+1}}\right)\right|_{n_{1}=n_{2}=0}\right\} .
\end{aligned}
$$

Since the fourth term in braces is equal to 0 , we have

$$
\begin{aligned}
\frac{\partial^{m+1}}{\partial s \partial z^{m}} \Phi_{\left(m_{1}, m_{2}\right)}(s, z, & \left.\left(\omega_{1}, \omega_{2}\right)\right)\left.\right|_{s=0} \\
& =-(m-1) !\left(V_{1}(z)+V_{2}(z)+V_{3}(z)\right)+C_{1}
\end{aligned}
$$

where $C_{1}$ is a constant and

$$
\begin{aligned}
V_{1}(z) & :=\sum_{n_{1}, n_{2} \geq 0} \varepsilon_{n_{1}, n_{2}}\left(\frac{n_{1}^{m_{1}-1} n_{2}^{m_{2}-1}}{\left(n_{1} \omega_{1}+n_{2} \omega_{2}+z\right)^{m}}-\frac{n_{1}^{m_{1}-1} n_{2}^{m_{2}-1}}{\left(n_{1} \omega_{1}+n_{2} \omega_{2}-z\right)^{m}}\right), \\
V_{2}(z) & :=\left.\frac{1}{2} \sum_{n_{1} \geq 0} \varepsilon_{n_{1}}\left(\frac{n_{1}^{m_{1}-1} n_{2}^{m_{2}-1}}{\left(n_{1} \omega_{1}+n_{2} \omega_{2}+z\right)^{m}}+\frac{n_{1}^{m_{1}-1} n_{2}^{m_{2}-1}}{\left(n_{1} \omega_{1}+n_{2} \omega_{2}-z\right)^{m}}\right)\right|_{n_{2}=0}, \\
V_{3}(z) & :=\left.\frac{1}{2} \sum_{n_{2} \geq 0} \varepsilon_{n_{2}}\left(\frac{n_{1}^{m_{1}-1} n_{2}^{m_{2}-1}}{\left(n_{1} \omega_{1}+n_{2} \omega_{2}+z\right)^{m}}+\frac{n_{1}^{m_{1}-1} n_{2}^{m_{2}-1}}{\left(n_{1} \omega_{1}+n_{2} \omega_{2}-z\right)^{m}}\right)\right|_{n_{1}=0} .
\end{aligned}
$$

We transform $V_{1}(z)$ by using Theorem 3 , and $V_{2}(z), V_{3}(z)$ by using the original Poisson summation formula. First we deal with $V_{1}(z)$. Assuming that $\operatorname{Im}(z)>0$ is fixed, we put

$$
H(t)=\frac{1}{(t+z)^{m}}-\frac{1}{(t-z)^{m}} .
$$


Then $H(t)$ satisfies conditions (T1)-(T3) of Theorem 3. For $x \geq 0$ the residue theorem gives

$$
\begin{aligned}
\widehat{H}(x) & =-2 \pi i \operatorname{Res}_{t=-z} H(t) e(-t x) \\
& =\frac{(-2 \pi i)^{m}}{(m-1) !} x^{m-1} e(x z) .
\end{aligned}
$$

By Lemma 6.1(1) we get

$$
\begin{aligned}
\widehat{H}^{(k)}(0) & =\lim _{x \downarrow 0} \widehat{H}^{(k)}(x) \\
& = \begin{cases}0 & \text { if } k=0,1, \ldots, m-2, \\
(-2 \pi i)^{m} & \text { if } k=m-1 .\end{cases}
\end{aligned}
$$

For $k=0, \ldots, m-1$ and $x>0$ we compute that

$$
\begin{aligned}
\widehat{H}^{(k)}(x) & =\frac{\partial^{k}}{\partial x^{k}}\left(2 \pi i \frac{(-1)^{m}}{(m-1) !} \frac{\partial^{m-1}}{\partial z^{m-1}}(e(x z))\right) \\
& =\frac{(-1)^{m}}{(m-1) !}(2 \pi i)^{k+1} \frac{\partial^{m-1}}{\partial z^{m-1}}\left(z^{k} e(x z)\right) .
\end{aligned}
$$

Hence Theorem 3 gives

$$
V_{1}(z)=-\frac{\pi i}{\omega_{1}^{m_{1}} \omega_{2}^{m_{2}}} \frac{\left(m_{1}-1\right) !\left(m_{2}-1\right) !}{(m-1) !}+\frac{d^{m-1}}{d z^{m-1}}\left(V I_{1}(z)+V I_{2}(z)\right)
$$

where

$$
\begin{aligned}
& V I_{1}(z):=-\frac{1}{(m-1) !} \frac{1}{\omega_{1}^{m_{1}} \omega_{2}^{m_{2}}} \sum_{k=0}^{m_{1}-1} \frac{(-1)^{k}}{(2 \pi i)^{m_{2}+k-1}}\left(\begin{array}{c}
m_{1}-1 \\
k
\end{array}\right)\left(m_{2}+k-1\right) ! \\
& \times z^{m_{1}-k-1} \sum_{n_{1}=1}^{\infty}\left(\sum_{n_{2}=0}^{\infty} \varepsilon_{n_{2}}\left(\frac{1}{\left(\frac{n_{1}}{\omega_{1}}-\frac{n_{2}}{\omega_{2}}\right)^{m_{2}+k}}+\frac{1}{\left(\frac{n_{1}}{\omega_{1}}+\frac{n_{2}}{\omega_{2}}\right)^{m_{2}+k}}\right)\right) e\left(\frac{n_{1}}{\omega_{1}} z\right), \\
& V I_{2}(z):=-\frac{1}{(m-1) !} \frac{1}{\omega_{1}^{m_{1}} \omega_{2}^{m_{2}}} \sum_{k=0}^{m_{2}-1} \frac{(-1)^{k}}{(2 \pi i)^{m_{1}+k-1}}\left(\begin{array}{c}
m_{2}-1 \\
k
\end{array}\right)\left(m_{1}+k-1\right) ! \\
& \quad \times z^{m_{2}-k-1} \sum_{n_{2}=1}^{\infty}\left(\sum_{n_{1}=0}^{\infty} \varepsilon_{n_{1}}\left(\frac{1}{\left(\frac{n_{2}}{\omega_{2}}-\frac{n_{1}}{\omega_{1}}\right)^{m_{1}+k}}+\frac{1}{\left(\frac{n_{2}}{\omega_{2}}+\frac{n_{1}}{\omega_{1}}\right)^{m_{1}+k}}\right)\right) e\left(\frac{n_{2}}{\omega_{2}} z\right) .
\end{aligned}
$$

We consider the sum over $n_{2}$ in $V I_{1}(z)$. Differentiating the well known formula

$$
\pi i\left(1+\frac{2}{e(w)-1}\right)=\pi \cot (\pi w)=\sum_{n=0}^{\infty} \varepsilon_{n}\left(\frac{1}{w+n}+\frac{1}{w-n}\right)
$$


$m_{2}+k-1$ times, we obtain

$$
\begin{aligned}
& \pi i \delta_{m_{2}+k-1}+2 \pi i \frac{d^{m_{2}+k-1}}{d w^{m_{2}+k-1}}\left(\frac{1}{e(w)-1}\right) \\
& \quad=(-1)^{m_{2}+k-1}\left(m_{2}+k-1\right) ! \sum_{n_{2}=0}^{\infty} \varepsilon_{n_{2}}\left(\frac{1}{\left(w+n_{2}\right)^{m_{2}+k}}+\frac{1}{\left(w-n_{2}\right)^{m_{2}+k}}\right),
\end{aligned}
$$

where $\delta_{k}=1$ if $k=0$ and $\delta_{k}=0$ if $k \in \mathbb{Z}_{\geq 1}$. Putting $w=n_{1} \omega_{2} / \omega_{1}$, we obtain

$$
\begin{aligned}
\sum_{n_{2}=0}^{\infty} \varepsilon_{n_{2}}\left(\frac{1}{\left(\frac{n_{1}}{\omega_{1}}-\frac{n_{2}}{\omega_{2}}\right)^{m_{2}+k}}+\frac{1}{\left(\frac{n_{1}}{\omega_{1}}+\frac{n_{2}}{\omega_{2}}\right)^{m_{2}+k}}\right) \\
=\frac{(-1)^{m_{2}+k-1} \omega_{2}^{m_{2}+k}}{\left(m_{2}+k-1\right) !} \\
\quad \times\left(\pi i \delta_{m_{2}+k-1}+\left.2 \pi i \frac{d^{m_{2}+k-1}}{d w^{m_{2}+k-1}}\left(\frac{1}{e(w)-1}\right)\right|_{w=n_{1} \omega_{2} / \omega_{1}}\right) .
\end{aligned}
$$

Applying this to $V I_{1}(z)$, we get

$$
\begin{aligned}
V I_{1}(z)= & \frac{\pi i}{(m-1) !} \frac{(-1)^{m_{2}}}{\omega_{1}^{m_{1}}}\left(\frac{\delta_{m_{2}-1}}{(2 \pi i)^{m_{2}-1}} z^{m_{1}-1} \sum_{n=1}^{\infty} e\left(\frac{n}{\omega_{1}} z\right)\right. \\
& +2 \sum_{k=0}^{m_{1}-1} \frac{\omega_{2}^{k}}{(2 \pi i)^{m_{2}+k-1}}\left(\begin{array}{c}
m_{1}-1 \\
k
\end{array}\right) z^{m_{1}-k-1} \\
& \left.\times\left.\sum_{n=1}^{\infty} \frac{d^{m_{2}+k-1}}{d w^{m_{2}+k-1}}\left(\frac{1}{e(w)-1}\right)\right|_{w=n \omega_{2} / \omega_{1}} e\left(\frac{n}{\omega_{1}} z\right)\right) .
\end{aligned}
$$

Next we deal with $V I_{2}(z)$. By symmetry, we obtain

$$
\begin{aligned}
V I_{2}(z)= & \frac{\pi i}{(m-1) !} \frac{(-1)^{m_{1}}}{\omega_{2}^{m_{2}}}\left(\frac{\delta_{m_{1}-1}}{(2 \pi i)^{m_{1}-1}} z^{m_{2}-1} \sum_{n=1}^{\infty} e\left(\frac{n}{\omega_{2}} z\right)\right. \\
& +2 \sum_{k=0}^{m_{2}-1} \frac{\omega_{1}^{k}}{(2 \pi i)^{m_{1}+k-1}}\left(\begin{array}{c}
m_{2}-1 \\
k
\end{array}\right) z^{m_{2}-k-1} \\
& \left.\times\left.\sum_{n=1}^{\infty} \frac{d^{m_{1}+k-1}}{d w^{m_{1}+k-1}}\left(\frac{1}{e(w)-1}\right)\right|_{w=n \omega_{1} / \omega_{2}} e\left(\frac{n}{\omega_{2}} z\right)\right) .
\end{aligned}
$$

Next we calculate $V_{2}(z)$. When $m_{2} \geq 2, V_{2}(z)=0$. We consider the case $m_{2}=1$ (that is, $m=m_{1}+1$ ). We put

$$
f(t)=t^{m_{1}-1}\left(\frac{1}{\left(t \omega_{1}+z\right)^{m}}+\frac{1}{\left(t \omega_{1}-z\right)^{m}}\right) .
$$


To apply the original Poisson summation formula, we calculate $\widehat{f}(x)$. For $x \geq 0$ the residue formula gives

$$
\begin{aligned}
\int_{-\infty}^{\infty}\left(\frac{1}{\left(t \omega_{1}+z\right)^{m}}+\frac{1}{\left(t \omega_{1}-z\right)^{m}}\right) & e(-t x) d t \\
& =-2 \pi i \operatorname{Res}_{t=-z / \omega_{1}} \frac{e(-t x)}{\left(t \omega_{1}+z\right)^{m}} \\
& =\frac{2 \pi i}{\omega_{1}} \frac{(-1)^{m}}{(m-1) !}\left(\frac{2 \pi i x}{\omega_{1}}\right)^{m-1} e\left(\frac{z}{\omega_{1}} x\right) \\
& =\frac{2 \pi i}{\omega_{1}} \frac{(-1)^{m}}{(m-1) !} \frac{\partial^{m-1}}{\partial z^{m-1}}\left(e\left(\frac{z}{\omega_{1}} x\right)\right) .
\end{aligned}
$$

Differentiating both sides $m_{1}-1$ times with respect to $x$, for $x>0$ we obtain

$$
\widehat{f}(x)=\frac{2 \pi i}{\omega_{1}^{m_{1}}} \frac{1}{(m-1) !} \frac{\partial^{m-1}}{\partial z^{m-1}}\left(z^{m_{1}-1} e\left(\frac{z}{\omega_{1}} x\right)\right) .
$$

Since both sides are continuous for $x \geq 0$, this equality is valid for $x \geq 0$. We also remark that $f(t)$ and $\widehat{f}(x)$ are even functions. Hence the Poisson summation formula gives

$$
\begin{aligned}
\sum_{n_{1}=0}^{\infty} \varepsilon_{n_{1}}\left(\frac{n_{1}^{m_{1}-1}}{\left(n_{1} \omega_{1}+z\right)^{m}}\right. & \left.+\frac{n_{1}^{m_{1}-1}}{\left(n_{1} \omega_{1}-z\right)^{m}}\right) \\
& =\frac{2 \pi i}{\omega_{1}^{m_{1}}} \frac{1}{(m-1) !} \frac{d^{m-1}}{d z^{m-1}}\left(z^{m_{1}-1} \sum_{n=1}^{\infty} e\left(\frac{n}{\omega_{1}} z\right)\right)
\end{aligned}
$$

Combining the above calculations in the cases $m_{2} \geq 2$ and $m_{2}=1$, we have

$$
\begin{aligned}
V_{2}(z)=-\frac{d^{m-1}}{d z^{m-1}}\left(\frac{\pi i}{(m-1) !}\right. & \frac{(-1)^{m_{2}}}{\omega_{1}^{m_{1}}} \\
& \left.\times \frac{\delta_{m_{2}-1}}{(2 \pi i)^{m_{2}-1}} z^{m_{1}-1} \sum_{n=1}^{\infty} e\left(\frac{n}{\omega_{1}} z\right)\right) .
\end{aligned}
$$

By symmetry, we obtain

$$
\begin{aligned}
V_{3}(z)=-\frac{d^{m-1}}{d z^{m-1}}\left(\frac{\pi i}{(m-1) !}\right. & \frac{(-1)^{m_{1}}}{\omega_{2}^{m_{2}}} \\
& \left.\times \frac{\delta_{m_{1}-1}}{(2 \pi i)^{m_{1}-1}} z^{m_{2}-1} \sum_{n=1}^{\infty} e\left(\frac{n}{\omega_{2}} z\right)\right) .
\end{aligned}
$$


It follows from (3.4)-(3.9) that

$$
\begin{aligned}
\frac{\partial^{m+1}}{\partial s \partial z^{m}} \Phi_{\left(m_{1}, m_{2}\right)} & \left.\left(s, z,\left(\omega_{1}, \omega_{2}\right)\right)\right|_{s=0} \\
= & -2 \pi i \frac{d^{m-1}}{d z^{m-1}}\left\{\frac{(-1)^{m_{2}}}{\omega_{1}^{m_{1}}} \sum_{k=0}^{m_{1}-1} \frac{\omega_{2}^{k}}{(2 \pi i)^{m_{2}+k-1}}\left(\begin{array}{c}
m_{1}-1 \\
k
\end{array}\right)\right. \\
& \times\left. z^{m_{1}-k-1} \sum_{n=1}^{\infty} \frac{d^{m_{2}+k-1}}{d w^{m_{2}+k-1}}\left(\frac{1}{e(w)-1}\right)\right|_{w=n \omega_{2} / \omega_{1}} e\left(\frac{n}{\omega_{1}} z\right) \\
& +\frac{(-1)^{m_{1}}}{\omega_{2}^{m_{2}}} \sum_{k=0}^{m_{2}-1} \frac{\omega_{1}^{k}}{(2 \pi i)^{m_{1}+k-1}}\left(\begin{array}{c}
m_{2}-1 \\
k
\end{array}\right) \\
& \left.\times\left. z^{m_{2}-k-1} \sum_{n=1}^{\infty} \frac{d^{m_{1}+k-1}}{d w^{m_{1}+k-1}}\left(\frac{1}{e(w)-1}\right)\right|_{w=n \omega_{1} / \omega_{2}} e\left(\frac{n}{\omega_{2}} z\right)\right\} \\
& +C_{2},
\end{aligned}
$$

where $C_{2}$ is a constant. Hence

$$
\begin{aligned}
&\left.\frac{\partial^{2}}{\partial s \partial z} \Phi_{\left(m_{1}, m_{2}\right)}\left(s, z,\left(\omega_{1}, \omega_{2}\right)\right)\right|_{s=0} \\
&=-2 \pi i\left\{\frac{(-1)^{m_{2}}}{\omega_{1}^{m_{1}} \omega_{2}^{m_{2}-1}} \sum_{k=0}^{m_{1}-1}\left(\begin{array}{c}
m_{1}-1 \\
k
\end{array}\right)\right. \\
& \quad \times\left.\sum_{n=1}^{\infty} \frac{d^{m_{2}+k-1}}{d w^{m_{2}+k-1}}\left(\frac{1}{e^{\omega_{2} w}-1}\right)\right|_{w=2 \pi i n / \omega_{1}} z^{m_{1}-k-1} e\left(\frac{n}{\omega_{1}} z\right) \\
&+\frac{(-1)^{m_{1}}}{\omega_{1}^{m_{1}-1} \omega_{2}^{m_{2}}} \sum_{k=0}^{m_{2}-1}\left(\begin{array}{c}
m_{2}-1 \\
k
\end{array}\right) \\
&\left.\times\left.\sum_{n=1}^{\infty} \frac{d^{m_{1}+k-1}}{d w^{m_{1}+k-1}}\left(\frac{1}{e^{\omega_{1} w}-1}\right)\right|_{w=2 \pi i n / \omega_{2}} z^{m_{2}-k-1} e\left(\frac{n}{\omega_{2}} z\right)\right\} \\
&+Q_{1}(z),
\end{aligned}
$$

where $Q_{1}(z)$ is a polynomial of degree at most $m-1$. It follows from

$$
z^{m_{1}-k-1} e\left(\frac{n}{\omega_{1}} z\right)=\left.\frac{\partial^{m_{1}-k-1}}{\partial w^{m_{1}-k-1}}\left(e^{w z}\right)\right|_{w=2 \pi i n / \omega_{1}}
$$

and the Leibniz rule that 


$$
\begin{aligned}
\left.\sum_{k=0}^{m_{1}-1}\left(\begin{array}{c}
m_{1}-1 \\
k
\end{array}\right) \sum_{n=1}^{\infty} \frac{d^{m_{2}+k-1}}{d w^{m_{2}+k-1}}\left(\frac{1}{e^{\omega_{2} w}-1}\right)\right|_{w=2 \pi i n / \omega_{1}} z^{m_{1}-k-1} e\left(\frac{n}{\omega_{1}} z\right) \\
=\left.\sum_{n=1}^{\infty} \frac{\partial^{m_{1}-1}}{\partial w^{m_{1}-1}}\left\{e^{w z} \frac{\partial^{m_{2}-1}}{\partial w^{m_{2}-1}}\left(\frac{1}{e^{\omega_{2} w}-1}\right)\right\}\right|_{w=2 \pi i n / \omega_{1}} \\
=\left.\frac{d}{d z} \sum_{n=1}^{\infty} \frac{\partial^{m_{1}-1}}{\partial w^{m_{1}-1}}\left\{\frac{e^{w z}}{w} \frac{\partial^{m_{2}-1}}{\partial w^{m_{2}-1}}\left(\frac{1}{e^{\omega_{2} w}-1}\right)\right\}\right|_{w=2 \pi i n / \omega_{1}}
\end{aligned}
$$

Applying this to (3.10), we obtain

$$
\begin{aligned}
& \left.\frac{\partial}{\partial s} \Phi_{\left(m_{1}, m_{2}\right)}\left(s, z,\left(\omega_{1}, \omega_{2}\right)\right)\right|_{s=0} \\
= & -2 \pi i\left\{\left.\frac{(-1)^{m_{2}}}{\omega_{1}^{m_{1}} \omega_{2}^{m_{2}-1}} \sum_{n=1}^{\infty} \frac{\partial^{m_{1}-1}}{\partial w^{m_{1}-1}}\left(\frac{e^{w z}}{w} \frac{\partial^{m_{2}-1}}{\partial w^{m_{2}-1}}\left(\frac{1}{e^{\omega_{2} w}-1}\right)\right)\right|_{w=2 \pi i n / \omega_{1}}\right. \\
+ & \left.\left.\frac{(-1)^{m_{1}}}{\omega_{1}^{m_{1}-1} \omega_{2}^{m_{2}}} \sum_{n=1}^{\infty} \frac{\partial^{m_{2}-1}}{\partial w^{m_{2}-1}}\left(\frac{e^{w z}}{w} \frac{\partial^{m_{1}-1}}{\partial w^{m_{1}-1}}\left(\frac{1}{e^{\omega_{1} w}-1}\right)\right)\right|_{w=2 \pi i n / \omega_{2}}\right\} \\
+ & Q_{2}(z)
\end{aligned}
$$

where $Q_{2}(z)$ is a polynomial of degree at most $m$.

Applying (3.11) to (3.3), we have

$$
\begin{aligned}
\left.\frac{\partial}{\partial s} \varphi_{r}(s, z, \underline{\omega})\right|_{s=0} & 2 \pi i(-1)^{r+1}\left\{\sum_{n=1}^{\infty} \sum_{m_{1}=0}^{M-1} \frac{a_{M, m_{1}}}{\omega_{1}^{m_{1}+1}}\right. \\
= & \left.\frac{\partial^{m_{1}}}{\partial w^{m_{1}}}\left(\frac{e^{w z}}{w} \sum_{m_{2}=0}^{N-1} \frac{(-1)^{m_{2}+1} a_{N, m_{2}}}{\omega_{2}^{m_{2}}} \frac{\partial^{m_{2}}}{\partial w^{m_{2}}}\left(\frac{1}{e^{\omega_{2} w}-1}\right)\right)\right|_{w=2 \pi i n / \omega_{1}} \\
& +\sum_{n=1}^{\infty} \sum_{m_{2}=0}^{N-1} \frac{a_{N, m_{2}}}{\omega_{2}^{m_{2}+1}} \\
& \left.\times\left.\frac{\partial^{m_{2}}}{\partial w^{m_{2}}}\left(\frac{e^{w z}}{w} \sum_{m_{1}=0}^{M-1} \frac{(-1)^{m_{1}+1} a_{M, m_{1}}}{\omega_{1}^{m_{1}}} \frac{\partial^{m_{1}}}{\partial w^{m_{1}}}\left(\frac{1}{e^{\omega_{1} w}-1}\right)\right)\right|_{w=2 \pi i n / \omega_{2}}\right\} \\
& +Q_{3}(z),
\end{aligned}
$$

where $Q_{3}(z)$ is a polynomial of degree at most $r$. Since

$$
g_{N}\left(-\frac{\partial}{\partial u}\right)\left(\frac{1}{e^{u}-1}\right)=\frac{1}{\left(e^{u}-1\right)^{N}},
$$


which can be easily proved by induction with respect to $N$, we obtain

$$
\sum_{m_{2}=0}^{N-1} \frac{(-1)^{m_{2}+1} a_{N, m_{2}}}{\omega_{2}^{m_{2}}} \frac{\partial^{m_{2}}}{\partial w^{m_{2}}}\left(\frac{1}{e^{\omega_{2} w}-1}\right)=-\frac{1}{\left(e^{\omega_{2} w}-1\right)^{N}} .
$$

Therefore

$$
\begin{aligned}
\left.\frac{\partial}{\partial s} \varphi_{r}(s, z, \underline{\omega})\right|_{s=0}= & 2 \pi i(-1)^{r}\left\{\left.\frac{1}{\omega_{1}} \sum_{n=1}^{\infty} g_{M}\left(\frac{1}{\omega_{1}} \frac{\partial}{\partial w}\right) P_{N}\left(w ; z ; \omega_{2}\right)\right|_{w=2 \pi i n / \omega_{1}}\right. \\
& \left.+\left.\frac{1}{\omega_{2}} \sum_{n=1}^{\infty} g_{N}\left(\frac{1}{\omega_{2}} \frac{\partial}{\partial w}\right) P_{M}\left(w ; z ; \omega_{1}\right)\right|_{w=2 \pi i n / \omega_{2}}\right\}+Q_{3}(z) .
\end{aligned}
$$

Applying this formula to (3.1) completes the proof.

4. Degeneration of multiple Euler products. In this section we prove Theorem 1. First, we show the relation between multiple sine functions and absolute tensor products of Hasse zeta functions for finite fields.

Lemma 4.1. Let $p_{1}, \ldots, p_{r}$ be prime numbers. Then

$$
\zeta\left(s, \mathbb{F}_{p_{1}}\right) \otimes \cdots \otimes \zeta\left(s, \mathbb{F}_{p_{r}}\right) \cong S_{r}\left(i s,\left(\frac{2 \pi}{\log p_{1}}, \ldots, \frac{2 \pi}{\log p_{r}}\right)\right)^{(-1)^{r}} .
$$

Proof. Since

$$
\zeta\left(s, \mathbb{F}_{p}\right) \cong \prod_{n \in \mathbb{Z}}\left(\left(s-\frac{2 \pi i n}{\log p}\right)\right)^{-1}
$$

we get

$$
\begin{aligned}
\zeta\left(s, \mathbb{F}_{p_{1}}\right) \otimes & \cdots \otimes \zeta\left(s, \mathbb{F}_{p_{r}}\right) \\
= & \prod_{n_{1}, \ldots, n_{r}=0}^{\infty}\left(\left(s-\frac{2 \pi i n_{1}}{\log p_{1}}-\cdots-\frac{2 \pi i n_{r}}{\log p_{r}}\right)\right)^{(-1)^{r}} \\
& \times \prod_{n_{1}, \ldots, n_{r}=1}^{\infty}\left(\left(s+\frac{2 \pi i n_{1}}{\log p_{1}}+\cdots+\frac{2 \pi i n_{r}}{\log p_{r}}\right)\right)^{-1} \\
\cong & s^{(-1)^{r}} \\
& \quad \prod_{\substack{n_{1}, \ldots, n_{r} \geq 0 \\
\left(n_{1}, \ldots, n_{r}\right) \neq(0, \ldots, 0)}} P_{r}\left(\frac{s}{2 \pi i\left(\frac{n_{1}}{\log p_{1}}+\cdots+\frac{n_{r}}{\log p_{r}}\right)}\right)^{(-1)^{r}} \\
& \quad \prod_{n_{1}, \ldots, n_{r} \geq 1} P_{r}\left(-\frac{s}{2 \pi i\left(\frac{n_{1}}{\log p_{1}}+\cdots+\frac{n_{r}}{\log p_{r}}\right)}\right)^{-1},
\end{aligned}
$$

where $P_{r}(u):=(1-u) \exp \left(u+u^{2} / 2+\cdots+u^{r} / r\right)$.

On the other hand, [KK2, Proposition 2.4] says that for $\underline{\omega}=\left(\omega_{1}, \ldots, \omega_{r}\right)$ $\in\left(\mathbb{R}_{>0}\right)^{r}$ we have 


$$
\begin{aligned}
S_{r}(z, \underline{\omega}) \cong z & \prod_{\substack{n_{1}, \ldots, n_{r} \geq 0 \\
\left(n_{1}, \ldots, n_{r}\right) \neq(0, \ldots, 0)}} P\left(-\frac{z}{n_{1} \omega_{1}+\cdots+n_{r} \omega_{r}}\right) \\
& \times \prod_{\substack{n_{1}, \ldots, n_{r} \geq 1 \\
n_{r}}} P_{r}\left(\frac{z}{n_{1} \omega_{1}+\cdots+n_{r} \omega_{r}}\right)^{(-1)^{r-1}} .
\end{aligned}
$$

Combining these completes the proof.

Proof of Theorem 1. Applying Theorem 2 with $z=i s, \omega_{1}=2 \pi / \log p$ and $\omega_{2}=2 \pi / \log q$ to Lemma 4.1 , we obtain the desired result.

5. Conjecture for a more general case. In this section, applying Theorem 2 and past studies $([\mathrm{K}],[\mathrm{KW}])$ about multiple sine functions we show that Conjectures 4 and 5 (see Section 1) are reasonable. First, we recall a property of multiple sine functions:

Lemma 5.1 ([KK2, Theorem 2.1(e)]). For $\underline{\omega} \in\left(\mathbb{R}_{>0}\right)^{r}$ and $c>0$,

$$
S_{r}(c z, c \underline{\omega})=S_{r}(z, \underline{\omega}) \text {. }
$$

The following proposition is the reason we expect Conjecture 4 to be true.

Proposition 5.2. (1.2) holds in the following cases:

(1) when $M_{1}=\cdots=M_{k}=1$ and $\underline{\omega}$ satisfies condition (ii) of Conjecture 4 ,

(2) when $k=1$ and $\underline{\omega}$ satisfies condition (i) of Conjecture 4,

(3) when $k=2$ and $\underline{\omega}$ satisfies condition (i) of Conjecture 4.

Proof. (1.2) holds in case (1) by [KW, Theorem 1.4(2)] and in case (3) by Theorem 2 .

Next, we handle case $(2)$. Let $\underline{\omega}=\left(\omega_{1}, \ldots, \omega_{1}\right) \in\left(\mathbb{R}_{>0}\right)^{r}$. It follows from Lemma 5.1, Lemma 4.1 and Theorem 1.2 that for $\operatorname{Im}(z)>0$ we have

$$
\begin{aligned}
S_{r}(z, \underline{\omega}) & =S_{r}\left(\frac{2 \pi z}{\omega_{1} \log 2},\left(\frac{2 \pi}{\log 2}, \ldots, \frac{2 \pi}{\log 2}\right)\right) \\
& \left.\cong\left(\zeta\left(s, \mathbb{F}_{2}\right)^{\otimes r}\right)^{(-1)^{r}}\right|_{s=2 \pi z /\left(i \omega_{1} \log 2\right)} \\
& \cong \exp \left[(-1)^{r} \sum_{l=1}^{r} \frac{1}{(-2 \pi i)^{l-1}} g_{r}^{(l-1)}\left(\frac{z}{\omega_{1}}\right) \operatorname{Li}_{l}\left(e^{2 \pi i z / \omega_{1}}\right)\right] .
\end{aligned}
$$

On the other hand, the right hand side of (1.2) is equal to

$$
\exp \left[\left.\frac{2 \pi i(-1)^{r}}{\omega_{1}} \sum_{n=1}^{\infty} g_{r}\left(\frac{1}{\omega_{1}} \frac{\partial}{\partial t}\right) \frac{e^{t z}}{t}\right|_{t=2 \pi i n / \omega_{1}}\right]
$$


Expanding $g_{r}$ and using the Leibniz rule, we have

$$
\begin{aligned}
g_{r}\left(\frac{1}{\omega_{1}} \frac{\partial}{\partial t}\right) \frac{e^{t z}}{t} & =\sum_{j=0}^{r-1} \frac{g_{r}^{(j)}(0)}{j !} \frac{1}{\omega_{1}^{j}} \frac{\partial^{j}}{\partial t^{j}}\left(\frac{e^{t z}}{t}\right) \\
& =\sum_{j=0}^{r-1} \frac{g_{r}^{(j)}(0)}{j !} \frac{1}{\omega_{1}^{j}} \sum_{l=0}^{j}\left(\begin{array}{l}
j \\
l
\end{array}\right) \frac{(-1)^{l} l !}{t^{l+1}} z^{j-l} e^{t z} \\
& =e^{t z} \sum_{l=0}^{r-1} \sum_{j=l}^{r-1}(-1)^{l} \frac{g_{r}^{(j)}(0)}{(j-l) !} \frac{z^{j-l}}{t^{l+1} \omega_{1}^{j}} \\
& =e^{t z} \sum_{l=0}^{r-1} \frac{(-1)^{l}}{t^{l+1} \omega_{1}^{l}} \sum_{j=0}^{r-1-l} \frac{g_{r}^{(j+l)}(0)}{j !}\left(\frac{z}{\omega_{1}}\right)^{j} \\
& =e^{t z} \sum_{l=0}^{r-1} \frac{(-1)^{l}}{t^{l+1} \omega_{1}^{l}} g_{r}^{(l)}\left(\frac{z}{\omega_{1}}\right) .
\end{aligned}
$$

Applying (5.3) to (5.2), we conclude that (5.2) equals (5.1).

From Lemma 4.1 we obtain the following result:

Proposition 5.3. If Conjecture 4 is true, then so is Conjecture 5.

\section{Appendix}

6.1. Fourier transformations. In this section we consider the differentiability and estimates for the Fourier transformation. Throughout this section suppose that $H$ satisfies (T1) and (T2) of Theorem 3.

LEMMA 6.1.

(1) $\widehat{H}$ is an $(m-1)$-times continuously differentiable function in $\mathbb{R}$.

(2) For any $k=0, \ldots, m-1$ we have

$$
\widehat{H}^{(k)}(x)=(-2 \pi i)^{k} \int_{-\infty}^{\infty} t^{k} H(t) e(-t x) d t .
$$

Proof. For any $k=0, \ldots, m-1$ it follows from (T2) that $t^{k} H(t)$ is absolutely integrable on $\mathbb{R}$. This implies the desired results.

LEMma 6.2. $\widehat{H}^{(k)}(x)=O\left(e^{-2 \pi A x}\right)$ for $k=0, \ldots, m-1$ and $x \geq 0$.

Proof. Applying Cauchy's theorem to (6.1), we have

$$
\begin{aligned}
\widehat{H}^{(k)}(x) & =(-2 \pi i)^{k} \int_{-\infty}^{\infty}(t-i A)^{k} H(t-i A) e(-(t-i A) x) d t \\
& =(-2 \pi i)^{k} e^{-2 \pi A x} \int_{-\infty}^{\infty}(t-i A)^{k} H(t-i A) e(-t x) d t .
\end{aligned}
$$


By (T2) the above integral is bounded uniformly for $x$. This completes the proof.

Lemma 6.3. Let $k=0, \ldots, m-1$. Then for $0<\alpha \leq A / 4$ and $x \geq 0$,

$$
\int_{-\infty}^{\infty} t^{k} h(2 \alpha+i t) e(-t x) d t=\frac{e^{4 \pi \alpha x}}{(-2 \pi i)^{k}} \widehat{H}^{(k)}(x)+O\left(\alpha e^{-\pi A x}\right) .
$$

Proof. By Cauchy's theorem and Lemma 6.1 we have

$$
\begin{aligned}
& \int_{-\infty}^{\infty} t^{k} h(2 \alpha+i t) e(-t x) d t=e^{4 \pi \alpha x} \int_{-\infty}^{\infty}(t+2 i \alpha)^{k} H(t) e(-t x) d t \\
& =e^{4 \pi \alpha x}\left(\frac{\widehat{H}^{(k)}(x)}{(-2 \pi i)^{k}}+\sum_{l=1}^{k}\left(\begin{array}{c}
k \\
l
\end{array}\right) \frac{(2 i \alpha)^{l}}{(-2 \pi i)^{k-l}} \widehat{H}^{(k-l)}(x)\right) .
\end{aligned}
$$

Applying Lemma 6.2 to the second term in brackets, we obtain the desired results.

6.2. Estimates for generic numbers. Throughout this section let $a$ be a fixed positive number such that $a$ and $a^{-1}$ are generic.

Lemma 6.4. Let $\varepsilon>0$ be fixed. Then for $n_{1}, n_{2} \in \mathbb{Z}_{>0}$,

$$
\left|n_{1} a-n_{2}\right|^{-1} \ll \min \left\{n_{2}^{-1} e^{\varepsilon n_{1}}, n_{1}^{-1} e^{\varepsilon n_{2}}\right\} .
$$

Proof. Since $a$ and $a^{-1}$ are generic, by [A, Lemma 2.4] we have

$$
\left|n_{1} a-n_{2}\right|^{-1} \ll n_{2}^{-1} e^{\varepsilon n_{1}}, \quad\left|n_{2} a^{-1}-n_{1}\right|^{-1} \ll n_{1}^{-1} e^{\varepsilon n_{2}} .
$$

These imply the desired result.

Lemma 6.5. Let $B>0$ and $\sigma>1$. Then

$$
\sum_{\substack{n_{1}, n_{2} \geq 0 \\\left(n_{1}, n_{2}\right) \neq(0,0)}} \frac{e^{-B n_{1}}}{\left|n_{1} a-n_{2}\right|^{\sigma}}<\infty
$$

Proof.

$$
\sum_{\substack{n_{1}, n_{2} \geq 0 \\\left(n_{1}, n_{2}\right) \neq(0,0)}} \frac{e^{-B n_{1}}}{\left|n_{1} a-n_{2}\right|^{\sigma}}=\frac{1}{a^{\sigma}} \sum_{n_{1}=1}^{\infty} \frac{e^{-B n_{1}}}{n_{1}^{\sigma}}+\sum_{n_{2}=1}^{\infty} \frac{1}{n_{2}^{\sigma}}+\sum_{n_{1}, n_{2} \geq 1} \frac{e^{-B n_{1}}}{\left|n_{1} a-n_{2}\right|^{\sigma}} .
$$

Clearly, the first and second terms converge. It follows from Lemma 6.4 that the third term is

$$
\sum_{n_{1}, n_{2} \geq 1} \frac{e^{-B n_{1}}}{\left|n_{1} a-n_{2}\right|^{\sigma}} \ll \sum_{n_{1}, n_{2} \geq 1} e^{-B n_{1}} \frac{e^{B n_{1} / 2}}{n_{2}^{\sigma}}<\infty .
$$


Lemma 6.6. Let $B_{1}, B_{2}>0$ and $\sigma \geq 1$ be fixed. Then as $\alpha \downarrow 0$ we have

$$
\sum_{\substack{\left.n_{1}, n_{2} \geq 0 \\ n_{1}, n_{2}\right) \neq(0,0)}} \frac{e^{-B_{1} n_{1}-\alpha B_{2} n_{2}}}{\left|n_{1} a-n_{2}\right|^{\sigma}} \ll \begin{cases}\log (1 / \alpha) & \text { if } \sigma=1, \\ 1 & \text { if } \sigma>1 .\end{cases}
$$

Proof. When $\sigma>1$, the inequality $e^{-\alpha B_{2} n_{2}} \leq 1$ for any $n_{2} \in \mathbb{Z}_{\geq 0}$ and Lemma 6.5 lead to the desired result. We consider the case $\sigma=1$. We have

$$
\begin{aligned}
& \sum_{\substack{n_{1}, n_{2} \geq 0 \\
n_{1}, n_{2} \neq \neq(0,0)}} \frac{e^{-B_{1} n_{1}-\alpha B_{2} n_{2}}}{\left|n_{1} a-n_{2}\right|} \\
& \quad=\frac{1}{a} \sum_{n_{1}=1}^{\infty} \frac{e^{-B_{1} n_{1}}}{n_{1}}+\sum_{n_{2}=1}^{\infty} \frac{e^{-\alpha B_{2} n_{2}}}{n_{2}}+\sum_{n_{1}, n_{2} \geq 1} \frac{e^{-B_{1} n_{1}-\alpha B_{2} n_{2}}}{\left|n_{1} a-n_{2}\right|} .
\end{aligned}
$$

The first term is bounded uniformly with respect to $\alpha$. The second term is

$$
\sum_{n_{2}=1}^{\infty} \frac{e^{-\alpha B_{2} n_{2}}}{n_{2}}=-\log \left(1-e^{-B_{2} \alpha}\right) \ll \log (1 / \alpha)
$$

From Lemma 6.4 the third term is

$$
\sum_{n_{1}, n_{2} \geq 1} \frac{e^{-B_{1} n_{1}-\alpha B_{2} n_{2}}}{\left|n_{1} a-n_{2}\right|} \ll \sum_{n_{1}, n_{2} \geq 1} e^{-B_{1} n_{1}-\alpha B_{2} n_{2}} \frac{e^{\left(B_{1} n_{1}\right) / 2}}{n_{2}} \ll \log (1 / \alpha)
$$

\section{References}

[A] H. Akatsuka, Euler product expression of triple zeta functions, Internat. J. Math. 16 (2005), 111-136.

[B] A. Baker, Transcendental Number Theory, Cambridge Univ. Press, 1975.

[KK1] S. Koyama and N. Kurokawa, Multiple zeta functions: the double sine function and the signed double Poisson summation formula, Compos. Math. 140 (2004), 1176-1190.

[KK2] -, -, Multiple sine functions, Forum Math. 15 (2003), 839-876.

[K] N. Kurokawa, Multiple zeta functions: an example, Adv. Pure Math. 21 (1992), 219-226.

[KW] N. Kurokawa and M. Wakayama, Absolute tensor products, Int. Math. Res. Not. 2004, no. 5, 249-260.

[M] Y. Manin, Lectures on zeta functions and motives (according to Deninger and Kurokawa), Astérisque 228 (1995), 121-163.

Department of Mathematics

Tokyo Institute of Technology

2-12-1 Ookayama, Meguro-ku

Tokyo, 152-8550, Japan

E-mail: akatsuka@math.titech.ac.jp 